\title{
Human Bone Marrow Mesenchymal Stem Cell- Derived Exosomes Attenuate Blood-Spinal Cord Barrier Disruption via the TIMP2/MMP Pathway after Acute Spinal Cord Injury
}

\section{Wang Xin ( $\nabla$ wxmedicine@163.com )}

Shanghai Sixth Peoples Hospital https://orcid.org/0000-0002-0958-9195

\section{Shi Qiang}

Shanghai 6th Peoples Hospital Affiliated to Shanghai Jiaotong University School of Medicine

\section{Ding Jianing}

Shanghai 6th Peoples Hospital Affiliated to Shanghai Jiaotong University School of Medicine

\section{Liang Jiaming}

Shanghai 6th Peoples Hospital Affiliated to Shanghai Jiaotong University School of Medicine

\section{Lin Fangqi}

Shanghai 6th Peoples Hospital Affiliated to Shanghai Jiaotong University School of Medicine Cai Bin

Shanghai 6th Peoples Hospital Affiliated to Shanghai Jiaotong University School of Medicine

\section{Chen Yuanyuan}

Shanghai 6th Peoples Hospital Affiliated to Shanghai Jiaotong University School of Medicine

\section{Zhang Guowang}

Shanghai 6th Peoples Hospital Affiliated to Shanghai Jiaotong University School of Medicine

\section{Xu Jianguang}

Shanghai 6th Peoples Hospital Affiliated to Shanghai Jiaotong University School of Medicine

\section{Lian Xiaofeng}

Shanghai 6th Peoples Hospital Affiliated to Shanghai Jiaotong University School of Medicine

\section{Research Article}

Keywords: Exosomes, Bone marrow mesenchymal stem cells, Tissue inhibitors of matrix metalloproteinases 2, Blood spinal cord barrier, Spinal cord injury

Posted Date: July 8th, 2021

DOI: https://doi.org/10.21203/rs.3.rs-667955/v1 
License: (c) (i) This work is licensed under a Creative Commons Attribution 4.0 International License. Read Full License 


\section{Abstract}

After spinal cord injury (SCl), destruction of the blood spinal cord barrier (BSCB) results in infiltration of blood cells, such as neutrophils and macrophages, leading to permanent neurological dysfunction. Previous studies have shown that human bone marrow mesenchymal stem cell (BMSC)-derived exosomes have a beneficial neuroprotective effect in SCI models. However, whether BMSC-Exos contribute to the integrity of the BSCB has not been clarified. The purpose of this study was to investigate the mechanism of BMSC-Exo-induced changes in the permeability of the BSCB after SCl. Here, we showed that BMSC-Exos can inhibit BSCB permeability damage and improve spontaneous repair in a SCI model. Importantly, tissue inhibitors of matrix metalloproteinases 2 (TIMP2) were shown to play an important role in the functions of BMSCS-Exos by inhibiting the matrix metalloproteinase (MMP) pathway, thereby mitigating the reduction in cell junction proteins. Moreover, the ability of BMSC-Exos was significantly attenuated when TIMP2 was inhibited by siRNA. This proof-of-principle study demonstrates that BMSC-Exos can preserve the integrity of the BSCB and improve functional recovery after SCl through the TIMP2/MMP signaling pathway.

\section{Introduction}

Spinal cord injury $(\mathrm{SCl})$ is a serious health problem that usually leads to permanent disability and causes a cascade of events ${ }^{[1]}$. SCI not only results in physical and psychological harm to patients but may also represent a huge economic burden for their families and society ${ }^{[2]}$. The blood spinal cord barrier (BSCB) plays an important role in the recovery of central nervous system diseases after $\mathrm{SCl}{ }^{[3]}$. It can restrict and regulate the entry of external substances into the central nervous system and maintain the homeostasis of the microenvironment and has a considerable effect on the pathophysiological process of various neurological diseases ${ }^{[4]}$. Previous $\mathrm{SCl}$ research has mainly focused on improving sensory and motor functions ${ }^{[5][6]}$. Recently, the treatment mechanism of BSCB after SCl has become a hot spot in neuroscience research. Similar to the blood-brain barrier, the BSCB is a special structure composed of endothelial cells, a basement membrane, pericytes and astrocytes connected by various tight junction proteins and adhesion junction proteins ${ }^{[7]}$. The destruction of the BSCB leads to inflammatory infiltration and produces neurotoxic products that disrupt the function of neurons and synapses, which results in "programmed death" of glia and neurons, thus causing permanent neurological deficits $^{[8]}$. Furthermore, the disruption of the BSCB is correlated with increased mortality after intravascular therapy, and the restoration of BSCB function can significantly diminish secondary nerve injury ${ }^{[9]}$. In summary, early recovery of the BSCB plays a vital role in the treatment of SCI.

Exosomes are a main subclass of extracellular vesicles that can be released into the extracellular environment, and they are secreted by almost all types of cells and exist widely in body fluids ${ }^{[10][11]}$. Bone marrow mesenchymal stem cells (BMSCs) have entered the clinical stage of SCl treatment. Exosomes derived from BMSCs are easier to obtain and store and subject to limited ethical restrictions compared 
potential efficacy of functional behavior recovery in $\mathrm{SCl}$ rats ${ }^{[13]}$. Research reports have indicated that the therapeutic effect of exosomes derived from BMSCs occurs via the NF-KB p65 pathway, which inhibits the migration of pericytes, thereby maintaining the integrity of the BSCB after $\mathrm{SCl}$ and leading to a reduction in neuronal cell apoptosis, axonal regeneration, and motor function ${ }^{[14]}$. In addition, exosomes derived from miR-544-overexpressing BMSCs improved functional recovery and promoted neuronal survival by attenuating inflammation after $\mathrm{SCl}^{[15]}$. Therefore, recent attention has focused on the use of BMSC-Exos to treat $\mathrm{SCl}$ because of their remarkable therapeutic efficacy.

Disruption of the BSCB by matrix metalloprotease (MMP) activation is a detrimental event that leads to blood cell infiltration, inflammation, and apoptosis, thereby contributing to permanent neurological disability after $\mathrm{SCI}^{[16]}$. One such control mechanism is the activity of tissue inhibitors of matrix metalloproteinases (TIMPs) ${ }^{[17]}$. TIMP2 is also involved in regulating cellular processes, such as cell proliferation, apoptosis and angiogenesis, through different mechanisms and is not related to MMP inhibition ${ }^{[18][19]}$. Hence, in this study, we examined the changes in the expression of MMP2, MMP9 and TIMP2 in SCl.

As a refractory disease, spinal cord injury currently has limited treatment methods and approaches. Bone marrow mesenchymal stem cell exosomes represent a new method for treating spinal cord injury; however, their mechanism and efficacy are not yet fully understood. Therefore, in this study, we examined TIMP2, a MMP inhibitor, to explore the role of BMSC-Exos in BSCB repair after SCI.

\section{Materials And Methods}

\subsection{Cell culture}

Human bone marrow mesenchymal stem cells (BMSCs; ScienCell) were cultured in MEM (Minimum Essential Medium) Alpha Medium (Corning) and 10\% fetal bovine serum (FBS, Gibco, NY, USA). BMSCs between passage 4 were used for subsequent experiments. Human brain microvascular endothelial cells (HBMECs) were purchased from ScienCell Research Laboratories (ScienCell Research Laboratories, San Diego, CA, USA). Cells were cultured in endothelial cell medium (ScienCell Research Laboratories) and incubated in a humidified atmosphere at $5 \% \mathrm{CO} 2$ and $37^{\circ} \mathrm{C}$.

\subsection{Inhibition of TIMP2}

After the harvest of BMSC-conditioned medium, BMSCs were divided into four groups and transfected with siRNA (small interfering RNA). Three siRNAs (siTIMP2 \#1, siTIMP2\#2 and siTIMP2 \#3) and the scramble control siRNA (Con siRNA) were obtained from Asia-Vector Biotechnology (Shanghai, China), which also performed virus packaging. Cell transfection was performed following the manufacturer's instructions. In brief, cells were inoculated in 24-well culture plates, $1 \times 10^{5}$ target cells were inoculated in each well, and the cells were cultured overnight. When the cell density was $30-50 \%, 50$ ul serum-free medium and $125 \mathrm{~m}$ siRNA were to a sterilized EP tube, mixed gently and incubated at room Loading [MathJax]/jax/output/CommonHTML/fonts/TeX/fontdata.js 
temperature for 5 minutes (the final concentration of siRNA was $50 \mathrm{nM}$ ). Then, in a sterilized EP tube, $3 \mu \mathrm{l}$ liposome transfection reagent (please refer to the corresponding transfection reagent manual) was dissolved in $50 \mu \mathrm{l}$ serum-free medium, mixed gently and incubated for $5 \mathrm{~min}$ at room temperature. SiRNA and liposome solution were gently mixed and incubated for 10-20 min at room temperature. The mixed solution was added to the 24-well plate cells, and the cells were collected for 24-72 hours for determination. The siRNA sequences used in this study were as follows: siTIMP2\#1: GAGATCAAGCAGATAAAGA; siTIMP2\#2: GGAAAGAAGGAATATCTCA; and siTIMP2\#3: GGAAGTGGACTCTGGAAAC.

\subsection{Real-time PCR}

Four groups of BMSCs were tested by PCR after transfection. TRIzol reagent was used to isolate total RNA from cells, while exosomal miRNAs were isolated using an Exosome RNA Purification Kit (Qiagen). For the miRNA analysis, reverse transcription reactions of miRNA were performed using a $4 \times$ Reverse Transcription Master Mix kit (EZBioscience). qRT-PCR miRNA analyses were carried out with FastStart Universal SYBR Green Master Mix (Roche). U6 and GAPDH were used as internal controls. The primer sequences were as follows: TIMP2 Forward 5- $G \forall C A T C \forall C G G G C A \mathbb{C} A G-3$ and Reverse 5 - $T \mathbb{C} C T \mathbb{C} A G \forall \mathbb{C} C A C \forall \mathbb{C}$ - 3; U6 Forward 5- CTCGC T CGGCAGCACA - 3 and Reverse 5 - $\forall C G C$ T CACG $\forall$ T TGCGT - 3; and GAPDH Forward 5-CTCTGA T TGGTCGTA T GGG - 3 and Reverse 5- TGG $\forall$ GATGGTGATGGGA T - 3.

\subsection{Exosome isolation}

BMSCs and siTIMP2-BMSCs were separated for the exosome analysis. After harvesting BMSC- and siTIMP2-BMSC-conditioned media, the cells were removed by centrifuging for $10 \mathrm{~min}$ at $500 \mathrm{~g}$. Next, the apoptosis vesicles and debris were removed by centrifugation of the supernatant for 20 min at 12,000 g and filtering through a $0.22 \mu \mathrm{m}$ filter. Then, ultracentrifugation was performed for 70 min at $110,000 \mathrm{~g}$ (Beckman Optima XPN, 45Ti) to collect the exosomes in the form of a pellet, which was further resuspended in phosphate-buffered saline (PBS) for further purification by ultracentrifugation for $70 \mathrm{~min}$ at $110,000 \mathrm{~g}$ to remove the contaminating protein. Finally, exosomes were stored at $-80^{\circ} \mathrm{C}$ after being resuspended in PBS. The protein content of the exosomes was determined by the Pierce BCA Protein Assay Kit (Thermo Fisher Scientific, USA).

\subsection{Exosome characterization and internalization}

The qNano platform (iZON Science, UK) was used to analyze the distribution and absolute size of the exosomes. Morphological examination of isolated exosomes was performed through transmission electron microscopy (TEM; HT 00, Hitachi, Japan). The expression of CD9, CD63, and TSG101 in exosomes was evaluated by Western blot analysis, and the following primary antibodies were used: CD9, CD63, and TSG101 (1:1000; rabbit IgG, Proteintech). A green fluorescent dye (DIO; Life Technologies) was utilized to label the exosomes in accordance with the manufacturer's instructions. Ultracentrifugation at 
labeled exosomes at a concentration of $50 \mu \mathrm{g} / \mathrm{ml}$ for 8 hours, followed by the use of 4,6-diamidino-2phenylindole (DAPl; Southern Biotech, Birmingham, AL, USA) to stain nuclei, which were then analyzed by fluorescence microscopy.

\subsection{Cell viability assay}

Cell viability was determined using the cell counting kit-8 (CCK-8) assay. HBMECs were plated into 96-well plates and treated with endothelial cell medium. After $1,2,3,4$, and 5 days of incubation, $10 \mu$ l CCK-8 reagent (Dojindo, Japan) was added to the culture medium and the absorbance of each well was observed at $450 \mathrm{~nm}$ by a microplate reader (Bio-Rad 680, Hercules, USA).

\subsection{Spinal cord injury}

Adult female Sprague-Dawley rats (220-250 g) were obtained from the Animal Room of Shanghai Sixth People's Hospital. All animal experiments conformed to the Guide for the Care and Use of Laboratory Animals from the National Institutes of Health, and all procedures were approved by the Animal Research Committee of the Sixth People's Hospital at Shanghai Jiao Tong University. All animals were housed under standard temperature conditions with a $12 \mathrm{~h}$ light/dark cycle and provided food and water. Rats were anaesthetized with $10 \%$ chloral hydrate $(3.5 \mathrm{ml} / \mathrm{kg}$, i.p.), and a laminectomy was performed at the T9 level to expose the underlying cord without disrupting the dura. The exposed spinal cord was subjected to moderate contusion injury (150 kdyn force with no dwell time) using an Infinite Horizon Impact Device. The sham-operated group rats underwent a T9 laminectomy without contusion injury. Postoperative care included manual urinary bladder emptying every $12 \mathrm{~h}$ until the return of bladder function and the administration of cefazolin sodium ( $50 \mathrm{mg} / \mathrm{kg}$, i.p.).

\subsection{Exosome treatment}

BMSC exosomes and siTIMP2-BMSC exosomes were injected subcutaneously near the back wound after $\mathrm{SCl}$ and administered once a day for 1 week for behavioral tests or for the indicated time points for other experiments. The sham-operated group rats received no exosome treatment.

\subsection{Behavioral tests}

Functional deficits after $\mathrm{SCl}$ were examined as previously described ${ }^{[20]}$. The Basso, Beattie, and Bresnahan (BBB) scores were assessed on an open field scale by two blinded independent examiners at $1,3,5,7$, and 14 days postoperation. Briefly, the BBB locomotion rating scale scores ranged from 0 points (complete paralysis) to 21 points (normal locomotion). The scale was based on the natural progression of locomotion recovery in rats with thoracic SCl.

\subsection{Western blot analysis}

For protein analysis, the protein extract was homogenized in modified RIPA buffer ( $50 \mathrm{mM}$ Tris- $\mathrm{HCl}, 1 \%$

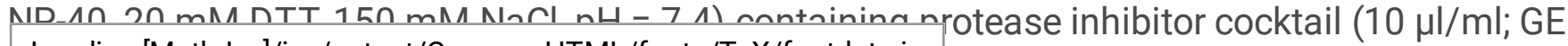
Loading [MathJax]/jax/output/CommonHTML/fonts/TeX/fontdata.js 
Healthcare Biosciences, PA, Little Chalfont, UK). The complex was then centrifuged at 11,792 g, and the supernatant was obtained for the protein assay. For protein analysis in vitro, HBMECs were lysed in RIPA buffer [25 mM Tris- $\mathrm{HCl}(\mathrm{pH} 7.6), 150 \mathrm{mM} \mathrm{NaCl}, 1 \%$ Nonidet P-40, $1 \%$ sodium deoxycholate and $0.1 \%$ SDS] with protease and phosphatase inhibitors. The extracts above were quantified with bicinchoninic acid reagents (Thermo, Rockford, IL, USA). Equivalent amounts of $50 \mu \mathrm{g}$ protein were separated using a $12 \%$ gel and then transferred onto a PVDF membrane (Bio-Rad, Hercules, CA, USA). The membrane was blocked with $5 \%$ nonfat milk in TBS with $0.05 \%$ Tween 20 for $1 \mathrm{~h}$ and then incubated with the following antibody solutions: MMP-2, MMP-9, beta-catenin, occludin, claudin-5, zo-1, and GAPDH. The membranes were washed with TBS three times and incubated with secondary antibodies for $2 \mathrm{~h}$ at room temperature. Signals were visualized using the ChemiDicTM XRS + Imaging System (Bio-Rad), and band densities were quantified with ImageJ software. The results are expressed as a relative density ratio normalized to the value of the Sham or Control group. Anti-beta-catenin, occludin, claudin-5, zo-1, and GAPDH were purchased from Abcam (Cambridge, UK).

\subsection{Hematoxylin and eosin staining}

The rats were anesthetized with $10 \%$ chloral hydrate $(3.5 \mathrm{ml} / \mathrm{kg}$, i.p.) and then perfused with $0.9 \% \mathrm{NaCl}$, followed by $4 \%$ paraformaldehyde in $0.01 \mathrm{M} \mathrm{PBS}(\mathrm{pH}=7.4)$ at 7 days after surgery. The spinal cords from the T7-T10 level around the lesion epicenter were excised, and transverse paraffin sections ( $5 \mathrm{~mm}$ thick) were mounted on poly-L-lysine-coated slides for histopathological examination by hematoxylin and eosin staining.

\subsection{Immunofluorescence staining}

The sections were incubated with $5 \%$ bovine serum albumin (BSA) for $1 \mathrm{~h}$ at room temperature and then incubated overnight at $4^{\circ} \mathrm{C}$ with primary antibodies in blocking buffer (zo-1, occludin, MMP-2, and MMP-9, Santa Cruz Biotechnology). Then, the cords were separately incubated with secondary antibody (Alexa Fluor 488-conjugated anti-IgG, Abcam; Texas red-conjugated anti-IgG, Santa Cruz Biotechnology). The nuclei were stained with DAPI (Beyotime Institute of Biotechnology, Shanghai, China). Cells grown on $14 \times 14 \mathrm{~mm}$ microscopic glass were washed with ice-cold PBS, fixed with $4 \%$ paraformaldehyde for 30 min, washed with ice-cold PBS, and blocked in $5 \%$ BSA for $1 \mathrm{hr}$. Then, the cells were incubated with antizo-1 (Abcam) and anti-Occludin (Abcam) diluted in 1\% BSA at $4^{\circ} \mathrm{C}$ overnight. Cells were washed with PBS followed by incubation with Alexa Fluor 488-conjugated anti-IgG or Texas red-conjugated anti-lgG secondary antibodies for $1 \mathrm{hr}$ at room temperature. After washing with PBS, the nuclei were stained with DAPI for 7 min and washed with PBS. Finally, Antifade Mounting Medium (Beyotime Institute of Biotechnology) was added to the cells.

\subsection{Evaluation of BSCB permeability}

2.13.1 Evans blue dye assays 
At 1 day after $\mathrm{SCl}$, the rats were intravenously injected with $2 \%$ Evans blue dye (EB; Sigma-Aldrich, 2 $\mathrm{ml} / \mathrm{kg}$ ) solution in saline into the tail vein. Two hours after injection, the rats were anesthetized with $10 \%$ chloral hydrate $(3.5 \mathrm{ml} / \mathrm{kg}$, i.p.) and then perfused with $0.9 \%$ normal saline. The injured spinal cord tissues of EB were weighed and immersed in N,NO-dimethylforma-mide (Shanghai, China) at $50^{\circ} \mathrm{C}$ for 72 h. The optical density of the supernatant was examined with an enzyme-labeled meter (at an excitation wavelength of $620 \mathrm{~nm}$ and an emission wavelength of $680 \mathrm{~nm}$ ). Dye in the samples was determined as $\mathrm{lg} / \mathrm{g}$ of tissue from a standard curve plotted using known amounts of dy $\mathrm{e}^{[21]}$.

\subsubsection{FITC-dextran assays}

At 1 day after $\mathrm{SCl}$, the rats were injected with 2\% FITC-dextran (MW 70 kDa, 4 mg/kg; Sigma-Aldrich) solution in PBS intravenously into the tail vein. Two hours after injection, the rats were anesthetized with $10 \%$ chloral hydrate $(3.5 \mathrm{ml} / \mathrm{kg}$, i.p.) and then perfused with $0.9 \%$ normal saline. The injured spinal cord tissues with FITC-dextran were weighed and homogenized in PBS and centrifuged. The optical density of the supernatant was examined (at an excitation wavelength of $493 \mathrm{~nm}$ and an emission wavelength of $517 \mathrm{~nm})$.

\subsubsection{Paracellular permeability assay}

Human brain microvascular endothelial cells were seeded on Transwell permeable supports (PET membrane 24-well cell culture inserts with $0.4 \mu \mathrm{m}$ pore size; Corelle; Corning Life Sciences, Corning, New York, USA) at a density of $1 \times 10^{5}$ cells/well in $200 \mu$ medium overnight and subjected to oxygen-glucose deprivation (OGD) for $10 \mathrm{~h}$. Then, the cells were incubated with FITC-dextran $(1 \mathrm{mg} / \mathrm{ml})$ in medium for another $2 \mathrm{~h}$ OGD. Thereafter, FITC-dextran passing through the Transwell (in the lower chambers) was determined by using an enzyme-labeled meter at an excitation wavelength of $493 \mathrm{~nm}$ and an emission wavelength of $517 \mathrm{~nm}$.

\subsection{Statistical analysis}

Means \pm SEM were used to express the data. Student's t-test was used to perform single comparisons, and one-way analysis of variance (ANOVA) was used to perform multiple comparisons. A value of $P$ $<0.05$ was used as a benchmark for statistical significance.

\section{Results}

\subsection{BMSC-Exos promotes SCI repair}

A qNano analysis was performed to identify BMSC-Exos, and the size distribution of most exosomes in the two groups was in the range of 50-150 nm (Fig. 1A), which was expected. The presence of exosomal marker proteins CD9, CD63, and TSG101 was detected in BMSC-Exos (Fig. 1B). These vesicles with characteristic cup-shaped morphology were observed via TEM (Fig. 1C). After SCl, the rats were immediatelv treated with Exos and further treated once a day for 1 week. Functional recovery was then Loading [MathJax]/jax/output/CommonHTML/fonts/TeX/fontdata.js 
estimated using BBB scores for locomotion. The exosome treatment significantly increased locomotor activity 3-14 days after injury compared with that observed in the $\mathrm{SCl}$ group (Fig. 1D). In addition, the HE staining method was used to stain SCl tissue sections. The results showed that the spinal cord tissue of the Sham group was dense and evenly distributed and had round and large nuclei, clear nucleoli, and no obvious apoptosis or necrosis. In the $\mathrm{SCl}$ group, bleeding was seen in the injured area and adjacent areas, some cells were lysed and disappeared, vacuoles were formed locally, the gray matter center was obviously necrotic, and the axonal disorder was irregular (Fig. 1E). The NeuN staining results also showed that neurons in the SCI group were significantly lost. After treatment with BMSC-Exos, the histopathological structure was significantly improved and neuron loss was reduced. The number of neurons increased significantly after treatment (Fig. 1F and G). The above data show that BMSC-Exos have a therapeutic effect on $\mathrm{SCl}$ in rats.

\subsection{BMSC-Exos attenuate BSCB disruption to promote SCI repair}

To detect whether BMSC-Exos have a protective effect on the integrity of the BSCB, Evans blue and FITCdextran fluorescence detection were used in this experiment. The fluorescence of Evans blue in the injured spinal cord (at 1 day) was higher than that in the sham group, and BMSC-Exos significantly reduced the fluorescence intensity, which can also be seen in the image of the spinal cord (Fig. 2A and B). In addition, BMSC-Exos significantly reduced the penetration of FITC-dextran (Fig. 2C). The above data indicate that BMSC-Exos attenuate BSCB disruption after SCl in rats.

\subsection{TIMP2 is involved in the effects of BMSC-Exos on SCI repair}

To investigate the role of TIMP2 in the cell junction repair effects of BMSCs-Exos on the BSCB after SCl, three siRNAs (siTIMP2 \#1, siTIMP2 \#2 and siTIMP2 \#3) were used to knock down the expression of TIMP2 in BMSCs, and the inhibitory efficiency of these siRNAs was tested by qRT-PCR (Fig. 3A). BMSCs transfected with the siTIMP2 \#2 siRNA, which showed the highest inhibitory efficiency, or with the scramble control siRNA (Con siRNA) were used as a "factory" to generate exosomes for downstream assays. The results of Western blotting showed that TIMP2 was downregulated in exosomes from siTIMP2-BMSCs (siTIMP2-Exos) (Fig. 3B and C). Subsequently, the rats were immediately treated with BMSC-Exos and siTIMP2-Exos after SCl and further treated once a day for 1 week. Functional recovery was also estimated using BBB scores for locomotion. Compared with the SCl group, the BMSCExo treatment significantly increased locomotor activity 3-14 days after injury. However, after removing the effect of TIMP2, the therapeutic effect of exosomes decreased (Fig. 3D), and this finding was consistent with the HE staining results of spinal cord tissue, which showed that the therapeutic effect of exosomes was significantly weakened (Fig. 3E). NeuN staining also demonstrated that si-TIMP2 inhibited the therapeutic effect of BMSC-Exos, and the number of neurons dropped significantly after TIMP2 was knocked out. (Fig. 4A and B). Evans blue dye extravasation was examined at $24 \mathrm{~h}$ after SCl. The results illustrated that si-TIMP2 reversed the protection caused by BMSC-Exos, as shown by the results of the Evans blue dye (Fig. 4C) and Evans blue extravasation assays (Fig. 4D). In addition, the penetration of 
FITC-dextran increased after TIMP2 was reduced (Fig. 4E). The above data show that BMSCExos improve functional recovery and attenuate BSCB disruption after SCI through TIMP2.

\subsection{BMSC-Exos increase the expression of junction proteins after SCI via the TIMP2/MMP pathway}

To test whether TIMP2 in BMSC-Exos can protect the integrity of the blood-spinal cord barrier by regulating tight junction proteins and adhesion junction proteins. We performed a Western blot analysis and immunofluorescence staining. First, TIMP2 decreased significantly in the SCI group and siTIMP2Exos group but increased in the Exos group (Fig. 5A and B), indicating that TIMP2 in exosomes entered the spinal cord injury site and that siRNA transfection was successful. TIMP2 affects the recovery of BSCB after SCl by inhibiting the MMP pathway, which leads to increased MMP-2 and MMP-9 as TIMP2 decreases in the corresponding group (Fig. 5A and C). Among them, the change in MMP-2 is greater than that of MMP-9, which also proves that TIMP2 has a greater impact on MMP-2. The results also showed that after $\mathrm{SCl}$, the expression of claudin-5, occludin, zo-1 and $\beta$-catenin was significantly reduced, and these changes can be attenuated by BMSC-Exos. However, after removing TIMP2, the effect of BMSCExos decreased (Fig. 5A and D). The results of immunofluorescence staining also showed that MMP-2 and MMP-9 were significantly increased in the $\mathrm{SCl}$ group and decreased with the addition of exosomes in the Exos group. After the removal of TIMP2, the MMP content rose again (Fig. 6A and B). The above data show that BMSC-Exos increase the expression of cell junction proteins after SCI via the TIMP2/MMP pathway.

\subsection{Effects of BMSC-Exos on junction proteins are inhibited by siTIMP2 in endothelial cells after oxygen- glucose deprivation}

For the in vitro experiments, we first determined whether Exos or siTIMP2-Exos could be internalized into HBMECs and whether internalization is an essential condition for the transfer of protein via exosomes.

Exos or siTIMP2-Exos were labeled with green fluorescent dye (DIO) and then incubated with HBMECs for 8 hours. Fluorescence microscopy confirmed that DIO-labeled Exos and DIO-labeled siTIMP2-Exos had been transferred to the perinuclear region of HBMECs (Fig. 7A). First, the CCK-8 assay showed a decreased ability of siTIMP2-Exos to promote the proliferation of HBMECs when TIMP2 was knocked down in their parental BMSCs (Fig. 7B). To confirm the influence of siRNA and OGD on the integrity of HBMECs, FITC-dextran was added to the cells after OGD. The results showed that cell permeability was significantly enhanced after OGD and significantly reduced after exosome addition. After TIMP2 was knocked down, the permeability of the endothelial barrier was further increased under OGD conditions (Fig. 7C). The Western blot analysis showed that with the addition of exosomes, the contents of MMP-2 and MMP-9 were significantly lower than those in the SCI group and recovered after TIMP2 was knocked out (Fig. 7D and E). Immunofluorescence showed that occludin and zo-1 were reduced with the occurrence of $\mathrm{SCI}$ while Exos significantly repaired cell junctions. This therapeutic effect was significantly suppressed after TIMP2 was knocked out (Fig. 8A-C). This experiment shows that SiTIMP2 attenuates the protective effect of Exos on junction proteins in endothelial cells after oxygen-glucose deprivation. 


\section{Discussion}

In this study, we showed that BMSC-Exos alleviate neurological deficits by preventing the destruction of the BSCB in rats after SCI. Through siRNA transfection, we confirmed that TIMP2 in BMSC-Exos inhibits the MMP pathway, thereby alleviating the destruction of cell junctions after $\mathrm{SCl}$, which contributes to BSCB repair. Here, we describe the mechanism by which BMSC-Exos affect the integrity of the BSCB after SCl.

The integrity of the BSCB exerts an enormous function on maintaining the normal function of the spinal cord. In clinical practice and animal models, the destruction of the BSCB is usually the inevitable result of $\mathrm{SCl}^{[21]}$. After $\mathrm{SCl}$, the blood vessels at the injured site are immediately destroyed and the BSCB far away from the injured area is permanently destroyed ${ }^{[22]}$. Studies have shown that growth factors, such as NGF and FGF-2, can improve functional recovery after SCI ${ }^{[23][24]}$. However, there are few studies on BSCB and stem cell exosomes. MSCs secrete more exosomes than other cells ${ }^{[25]}$. MSC-derived exosomes, similar to general exosomes, carry a complex cargo, including proteins, nucleic acids, and lipids ${ }^{[26][27]}$. Yuan et al. ${ }^{[28]}$ directly used pericyte-derived exosomes to treat $\mathrm{SCl}$ and found that they reduce cell apoptosis, improve microcirculation in the spinal cord after injury, and prevent BSCB injury and edema. The exosomes of BMSCs play a significant role in $\mathrm{SCl}$ and have many effects on neuronal apoptosis, angiogenesis, inflammation, etc.; however, their function in BSCB repair has not been fully explained ${ }^{[29][30]}$. In this study, we demonstrated the protective effect of BMSC-Exos on the BSCB in vivo and in vitro, thereby promoting the recovery of neurological function after $\mathrm{SCl}$.

Crilger first proved that BMSCs can secrete brain-derived neurotrophic factor (BDNF) and $\beta$-nerve growth factor ( $\beta$-NGF) to promote neuron survival and axon regeneration in vitro ${ }^{[31]}$. A large number of studies have proven the safety and effectiveness of BMSC-Exos in the treatment of $\mathrm{SCl}$ in vivo or in vitro. Some studies have compared the effects of BMSCs and BMSC-Exos in the treatment of $\mathrm{SCl}$. Soluble components and BMSC-Exos have multiple functions, including neuroprotection, immune regulation, scar formation reduction, nerve regeneration promotion and blood vessel formation ${ }^{[32]}$. For example, insulin-like growth factor 1 (IGF-1), hepatocyte growth factor (HGF) and transforming growth factor $\beta$ (TGF- $\beta$ ) related to axon growth and nerve survival can be found in BMSC-Exos, which improve the local environment of $\mathrm{SCl}$ by reducing scar formation stem cell factor (SCF) and stromal cell-derived factor (SDF-1a) ${ }^{[31]}$. The pathological and regenerative mechanisms of $\mathrm{SCl}$ are complex, and it is difficult to achieve a satisfactory therapeutic effect on a single target ${ }^{[33]}$. BMSC-Exos played an important role in the BBB score, neuronal recovery and other indicators. In terms of BSCB repair, BMSC-Exos also have excellent performance. This study shows that BMSC-Exos can significantly reduce the expression of MMP, thereby repairing cell connections and further destroying the BSCB through TIMP2. It is worth noting that after using siRNA to reduce the expression of TIMP2 in BMSC-Exos, BMSC-Exos still express a certain $\mathrm{SCl}$ therapeutic effect, which has certain effects on the MMP, cell connexin and BSCB. Although the knockdown of TIMP2 significantly reduced the therapeutic effect of BMSC-Exos, the 
The expression and distribution of tight junction proteins are closely related to the permeability of the BSCB after SCl[ ${ }^{34]}$. After SCl, the expression of occludin and ZO-1 decreases, especially at 1 and 3 days after injury ${ }^{[35][36]}$. In the study by Wu et al. occludin, claudin- 5 and ZO-1 were lost at $48 \mathrm{~h}$ after injury, which aggravated the damage to BSCB integrity ${ }^{[37]}$. Our data also showed that the expression of occludin, claudin-5, ZO-1 and $\beta$-catenin decreased significantly after $\mathrm{SCl}$ and the destruction of cell connections is one of the mean reasons for BSCB damage after $\mathrm{SCl}$, in which the MMP pathway has an immense influence ${ }^{[38][39]}$. Our results found that after $\mathrm{SCl}$, the expression levels of MMP-2 and MMP-9 were upregulated, with MMP-2 showing more pronounced expression. TIMP2, as an inhibitor of MMP-2, enters the injured area in the BMSC-Exo load, thereby inhibiting MMP, repairing cell junctions, and preventing further destruction of the BSCB.

The upregulation of MMP can degrade the base components of tight junction structure and adhesion junction structure, thereby allowing the infiltration of immune cells and leading to secondary damage induced by $\mathrm{SCl}^{[40]}$. Wang et al. showed that valproic acid inhibits the destruction of the blood-brain barrier and cerebral edema caused by ischemia by inhibiting the induction of MMP and the destruction of tight junctions ${ }^{[41]}$. Researchers such as Lee also found that fluoxetine or valproic acid can inhibit the expression and activity of MMP-9 and prevent the destruction of the BSCB after SCI ${ }^{[3][36]}$. Research reports have indicated that the upregulation of MMP-2 after SCI leads to the early initial opening of the blood-brain barrier, which degrades the basement membrane and causes neuronal damage ${ }^{[42]}$. On the 5 th day after injury, MMP-2 was activated ${ }^{[36]}$. The results of in vitro experiments also showed that BMSCExos increase the expression of tight junction proteins and adhesion junction proteins after OGD but also reduce the expression of MMP after OGD. To further explore the role of TIMP2, after knocking out its expression in BMSC-Exos and detecting its downstream MMP, the expression of tight junction proteins and adhesion junction proteins showed obvious corresponding changes. The results of the experiment showed that BMSC-Exos have an obvious repair effect on the cell-to-cell connection after $\mathrm{SCl}$ and that TIMP2 plays an important role in this process. In addition, in vitro FITC-dextran penetration experiments also demonstrated the protective effect of BMSC-Exos on the endothelial barrier. Therefore, BMSC-Exos protect the integrity of the BSCB by reducing the activity of excessive MMP through TIMP2, which can be considered a therapeutic target for reducing secondary damage after SCl.

In conclusion, this study explored the protective effect of BMSC-Exos on the integrity of the BSCB after $\mathrm{SCl}$. Our research shows that BMSC-Exos attenuate the destruction of the BSCB and improve functional recovery after SCI through the TIMP2/MMP signaling pathway. These results indicate that TIMP2/MMP is a significant signaling pathway involved in the integrity of the BSCB. Our results suggest that BMSCExos may provide a potential therapeutic intervention for preventing BSCB rupture after SCl.

\section{Declarations}

Acknowledgements 
This work was supported by the National Natural Science

Foundation of China (NSFC 81272001).

Funding: This work was supported by the National Natural Science Foundation of China (NSFC 81272001).

Conflicts of interest: The authors declare that they have no competing interests.

Availability of data and material: Not applicable.

Code availability: Not applicable.

Authors' contributions: Author names:Wang Xin,MD\#; Shi Qiang,MD\#; Ding Jianing,MD\#; Liang Jiaming,MD; Lin Fangqi,MD; Cai Bin, MD; Chen Yuanyuan,MD; Zhang Guowang,MD; Xu Jianguang,MD; Lian Xiaofeng, MD*. \# These authors contributed equally to this work, all authors read and approved the final manuscript.

Ethics approval: All animal experiments conformed to the Guide for the Care and Use of Laboratory Animals from the National Institutes of Health, and all procedures were approved by the Animal Research Committee of the Sixth People's Hospital at Shanghai Jiao Tong University.

Consent to participate: Not applicable.

Consent for publication: All data generated or analysed during this study are included in this published article.

\section{References}

1. Whetstone WD, Hsu JY, Eisenberg M et al (2003) Blood-spinal cord barrier after spinal cord injury: relation to revascularization and wound healing. J Neurosci Res 74:227-239. https://doi.org/10.1002/jnr.10759

2. Muresanu DF, Sharma A, Lafuente JV et al (2015) Nanowired delivery of growth hormone attenuates pathophysiology of spinal cord injury and enhances insulin-like growth factor- 1 concentration in the plasma and the spinal cord. Mol Neurobiol 52:837-45.449. https://doi.org/10.1007/s12035-0159298-8

3. Bartanusz V, Jezova D, Alajajian B et al (2011) The blood-spinal cord barrier: morphology and clinical implications. Ann Neurol 70:194-206. https://doi.org/10.1002/ana.22421

4. Kaneko S, Iwanami A, Nakamura $M$ et al.,2006. A selective Sema3A inhibitor enhances regenerative responses and functional recovery of the injured spinal cord. Nature medicine. 12:1380-9

5. https://doi.org/10.1038/nm1505 
6. Mikami Y, Toda M, Watanabe M et al (2002) A simple and reliable behavioral analysis of locomotor function after spinal cord injury in mice. Technical note. Journal of neurosurgery 97:142-147. https://doi.org/10.3171/spi.2002.97.1.0142

7. Jeong SR, Kwon MJ, Lee HG et al.,2012. Hepatocyte growth factor reduces astrocytic scar formation and promotes axonal growth beyond glial scars after spinal cord injury. Exp Neurol. 233:312 - 22. https://doi.org/10.1016/j.expneurol.2011.10.021

8. Bartanusz V, Jezova D, Alajajian B et al (2011) The blood-spinal cord barrier: morphology and clinical implications. Annals of neurology 70:194-206. https://doi.org/10.1002/ana.22421

9. Sharma HS.,2011. Early microvascular reactions and blood-spinal cord barrier disruption are instrumental in pathophysiology of spinal cord injury and repair: novel therapeutic strategies including nanowired drug delivery to enhance neuroprotection. Journal of neural transmission 118:155 - 76. https://doi.org/10.1007/s00702-010-0514-4

10. Schnell L, Schneider R, Kolbeck R et al (1994) Neurotrophin-3 enhances sprouting of corticospinal tract during development and after adult spinal cord lesion. Nature 367:170-173. https://doi.org/10.1038/367170a0

11. Zeringer E, Barta T, Li M et al.,2015. Strategies for isolation of exosomes. Cold Spring Harb Protoc. 2015(4):319-23. https://doi.org/10.1101/pdb.top074476

12. Colombo M, Raposo G, Théry C (2014) Biogenesis, secretion, and intercellular interactions of exosomes and other extracellular vesicles. Annu Rev Cell Dev Biol 30:255-289. https://doi.org/10.1146/annurev-cellbio-101512-122326

13. Gimona M, Pachler K, Laner-Plamberger S et al (2017) Manufacturing of human extracellular vesiclebased therapeutics for clinical use. Int J Mol Sci 18(6):1190. https://doi.org/10.3390/ijms18061190

14. Yu L, Sui B, Fan W et al.,2021. Exosomes derived from osteogenic tumor activate osteoclast differentiation and concurrently inhibit osteogenesis by transferring COL1A1-targeting miRNA-92a-15p. J Extracell Vesicles Jan;103(3). https://doi.org/10.1002/jev2.12056

15. Lu Y, Zhou Y, Zhang R et al (2019) Bone Mesenchymal stem cell-derived extracellular vesicles promote recovery following spinal cord injury via improvement of the integrity of the blood-spinal cord barrier. Front Neurosci 13:209. https://doi.org/10.3389/fnins.2019.00209

16. Gu J, Jin ZS, Wang CM et al.,2020. Bone Marrow Mesenchymal Stem Cell-Derived Exosomes Improves Spinal Cord Function After Injury in Rats by Activating Autophagy. Drug Des Devel Ther.14. https://doi.org/10.3389/fnins.2019.00209

17. Li C, Li X, Zhao B et al.,2020. Exosomes derived from miR-544-modified mesenchymal stem cells promote recovery after spinal cord injury. Arch Physiol Biochem Oct;1264(4). https://doi.org/10.1080/13813455.2019.1691601

18. Lee JY, Na WH, Choi HY et al.,2016. Jmjd3 mediates blood-spinal cord barrier disruption after spinal cord injury by regulating MMP-3 and MMP-9 expressions. Neurobiol Dis. Nov;95. https://doi.org/10.1016/j.nbd.2016.07.015 
19. Wright JW, Harding JW.,2009. Contributions of matrix metalloproteinases to neural plasticity, habituation, associative learning and drug addiction. Neural Plast. 2009, 579382. https://doi.org/10.1155/2009/579382

20. Baker AH, Edwards DR, Murphy $G$ et al (2002) Metalloproteinase inhibitors: Biological actions and therapeutic opportunities. J Cell Sci 115 Pt 19:3719-3727. https://doi.org/10.1242/jcs.00063

21. Mannello F, Gazzanelli G (2001) Tissue inhibitors of metalloproteinases and programmed cell death: Conundrums, controversies and potential implications. Apoptosis 6:479-482. https://doi.org/10.1023/a:1012493808790

22. Bartanusz V, Jezova D, Alajajian B et al (2011) The blood-spinal cord barrier: morphology and clinical implications. Ann Neurol 70(2):194-206. https://doi.org/10.1002/ana.22421

23. Figley SA, Khosravi R, Legasto JM et al (2014) Characterization of vascular disruption and bloodspinal cord barrier permeability following traumatic spinal cord injury. J Neurotrauma 31(6):541552. https://doi.org/10.1089/neu.2013.3034

24. Zhang HY, Wang ZG, Wu FZ et al (2013) Regulation of autophagy and ubiquitinated protein accumulation by bFGF promotes functional recovery and neural protection in a rat model of spinal cord injury. Mol Neurobiol 48:452-464. https://doi.org/10.1007/s12035-013-8432-8

25. Chen X, Lan X, Roche I et al (2008) Caffeine protects against MPTP-induced blood-brain barrier dysfunction in mouse striatum. J Neurochem 107:1147-1157. https://doi.org/10.1111/j.14714159.2008.05697.x

26. Yu B, Zhang X, Li X (2014) Exosomes derived from mesenchymal stem cells. Int J Mol Sci 514 15(3):4142-4157. https://doi.org/10.3390/ijms15034142

27. Villarroya-Beltri C, Baixauli F, Gutiérrez-Vázquez C et al (2014) Sorting it out: regulation of exosome loading. Semin Cancer Biol 28:3-13. https://doi.org/10.1016/j.semcancer.2014.04.009

28. Vlassov AV, Magdaleno S, Setterquist R et al (2012) Exosomes: current knowledge of their composition, biological functions, and diagnostic and therapeutic potentials. Biochim Biophys Acta 1820(7):940-948. https://doi.org/10.1016/j.bbagen.2012.03.017

29. Yuan $X$, Wu Q, Wang P et al (2019) Exosomes derived from pericytes improve microcirculation and protect blood-spinal cord barrier after spinal cord injury in mice. Front Neurosci 13:319. https://doi.org/10.3389/fnins.2019.00319

30. Liu W, Wang Y, Gong F et al.,2019. Exosomes Derived from Bone Mesenchymal Stem Cells Repair Traumatic Spinal Cord Injury by Suppressing the Activation of A1 Neurotoxic Reactive Astrocytes. J Neurotrauma. 02 01;363(3). https://doi.org/10.1089/neu.2018.5835

31. Luo Y, Xu T, Liu W et al.,2021. Exosomes derived from GIT1-overexpressing bone marrow mesenchymal stem cells promote traumatic spinal cord injury recovery in a rat model. Int $\mathrm{J}$ Neurosci Feb;1312(2). https://doi.org/10.1080/00207454.2020.1734598

32. Teixeira FG, Carvalho MM, Sousa $\mathrm{N}$ et al (2013) Mesenchymal stem cells secretome: a new paradigm for central nervous system regeneration. Cell Mol Life Sci 70(20):3871-3882. 
33. Kanekiyo K, Wakabayashi T, Nakano N et al (2018) Effects of Intrathecal Injection of the Conditioned Medium from Bone Marrow Stromal Cells on Spinal Cord Injury in Rats. J Neurotrauma 35(3):521532. https://doi.org/10.1089/neu.2017.5201

34. Hutson TH, DI Giovanni S (2019) The translational landscape in spinal cord injury: focus on neuroplasticity and regeneration. Nat Rev Neurol 15(12):732-745. https://doi.org/10.1038/s41582019-0280-3

35. Liebner S, Czupalla CJ, Wolburg H,2011. Current concepts of blood-brain barrier development. The International journal of developmental biology 55:467 - 76. https://doi.org/10.1387/ijdb.103224sl

36. Lee JY, Kim HS, Choi HY et al (2012) Fluoxetine inhibits matrix metalloprotease activation and prevents disruption of blood-spinal cord barrier after spinal cord injury. Brain 135:2375-2389. https://doi.org/10.1093/brain/aws171

37. Lee JY, Kim HS, Choi HY et al.,2012. Valproic acid attenuates blood-spinal cord barrier disruption by inhibiting matrix metalloprotease-9 activity and improves functional recovery after spinal cord injury. Journal of neurochemistry 121:818 - 29. https://doi.org/10.1111/j.1471-4159.2012.07731.x

38. Wu Q, Jing Y, Yuan X et al.,2014. Melatonin Treatment Protects Against Acute Spinal Cord InjuryInduced Disruption of Blood Spinal Cord Barrier in Mice. Journal of molecular neuroscience: MN. https://doi.org/10.1007/s12031-014-0430-4

39. Asahi M, Wang X, Mori T et al (2001) Effects of matrix metalloproteinase-9 gene knock-out on the proteolysis of blood-brain barrier and white matter components after cerebral ischemia. The Journal of neuroscience: the official journal of the Society for Neuroscience 21:7724-7732. https://doi.org/10.1523/jneurosci.21-19-07724.2001

40. Jin YJ, Park I, Hong IK et al (2010) Fibronectin and vitronectin induce AP-1-mediated matrix metalloproteinase-9 expression through integrin $a(5) \beta(1) / a(v) \beta(3)$-dependent Akt, ERK and JNK signaling pathways in human umbilical vein endothelial cells. Cell Signal 23:125-134. https://doi.org/10.1016/j.cellsig.2010.08.012

41. Noble LJ, Donovan F, Igarashi T et al (2002) Matrix metalloproteinases limit functional recovery after spinal cord injury by modulation of early vascular events. The Journal of neuroscience 22:75267535. https://doi.org/10.1523/jneurosci.22-17-07526.2002

42. Wang Z, Leng Y, Tsai LK et al (2011) Valproic acid attenuates blood-brain barrier disruption in a rat model of transient focal cerebral ischemia: the roles of HDAC and MMP-9 inhibition. Journal of cerebral blood flow metabolism 31:52-57. https://doi.org/10.1038/jcbfm.2010.195

43. Dang AB, Tay BK, Kim HT et al (2008) Inhibition of MMP2/MMP9 after spinal cord trauma reduces apoptosis. Spine 33:E576-E579. https://doi.org/10.1097/BRS.0b013e31817ecc87

\section{Figures}



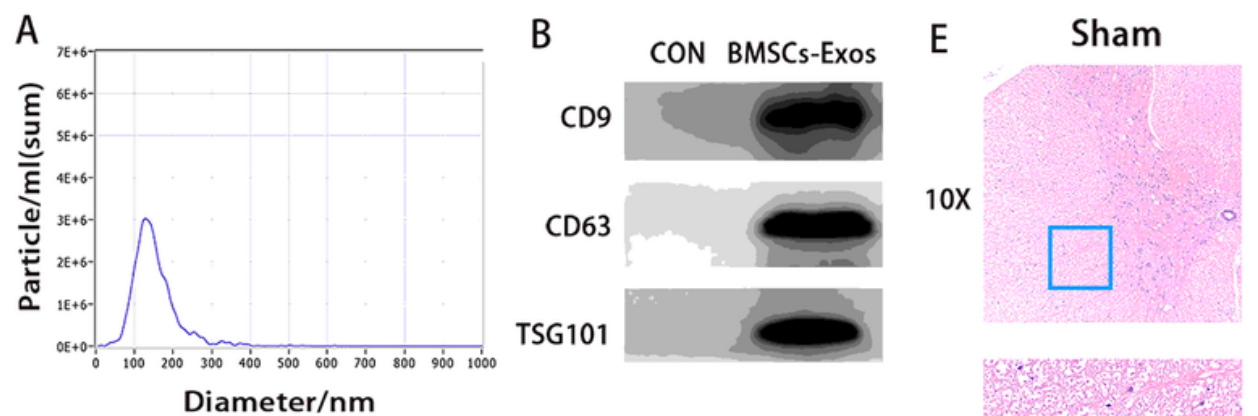

$\mathrm{SCl}$

Exos

C
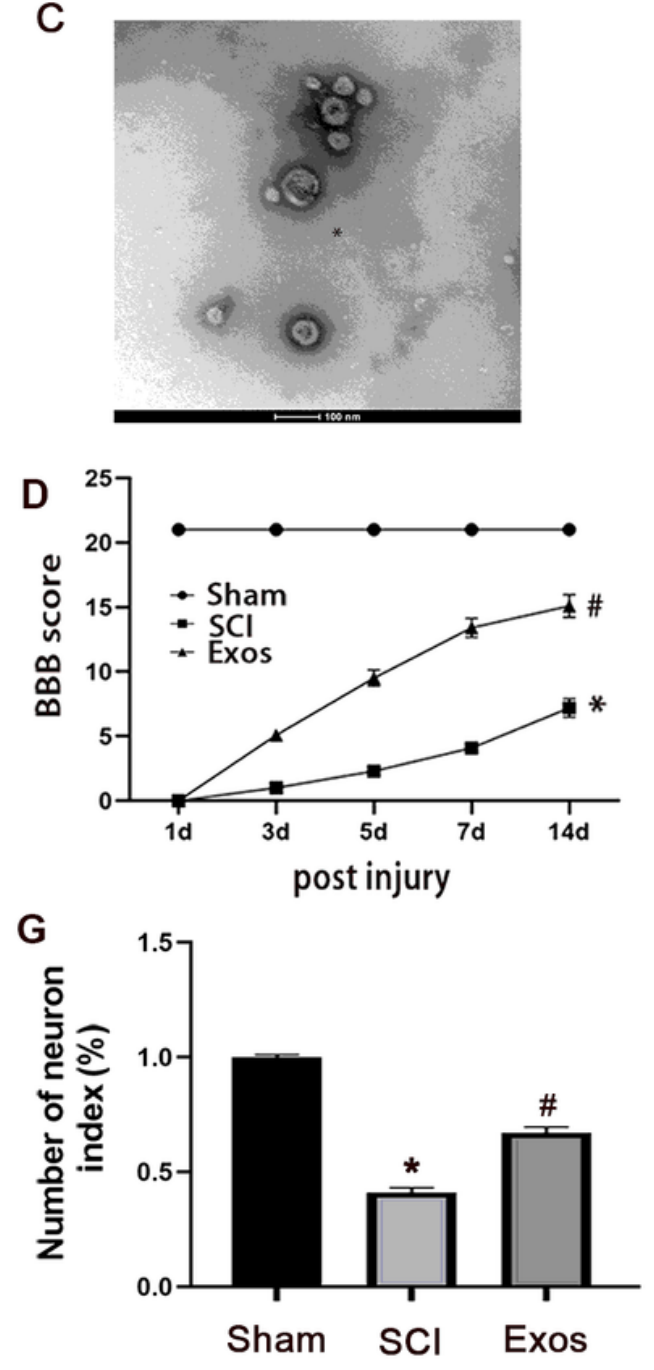

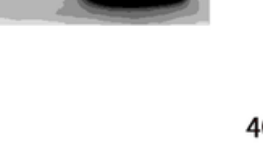

$40 \mathrm{X}$
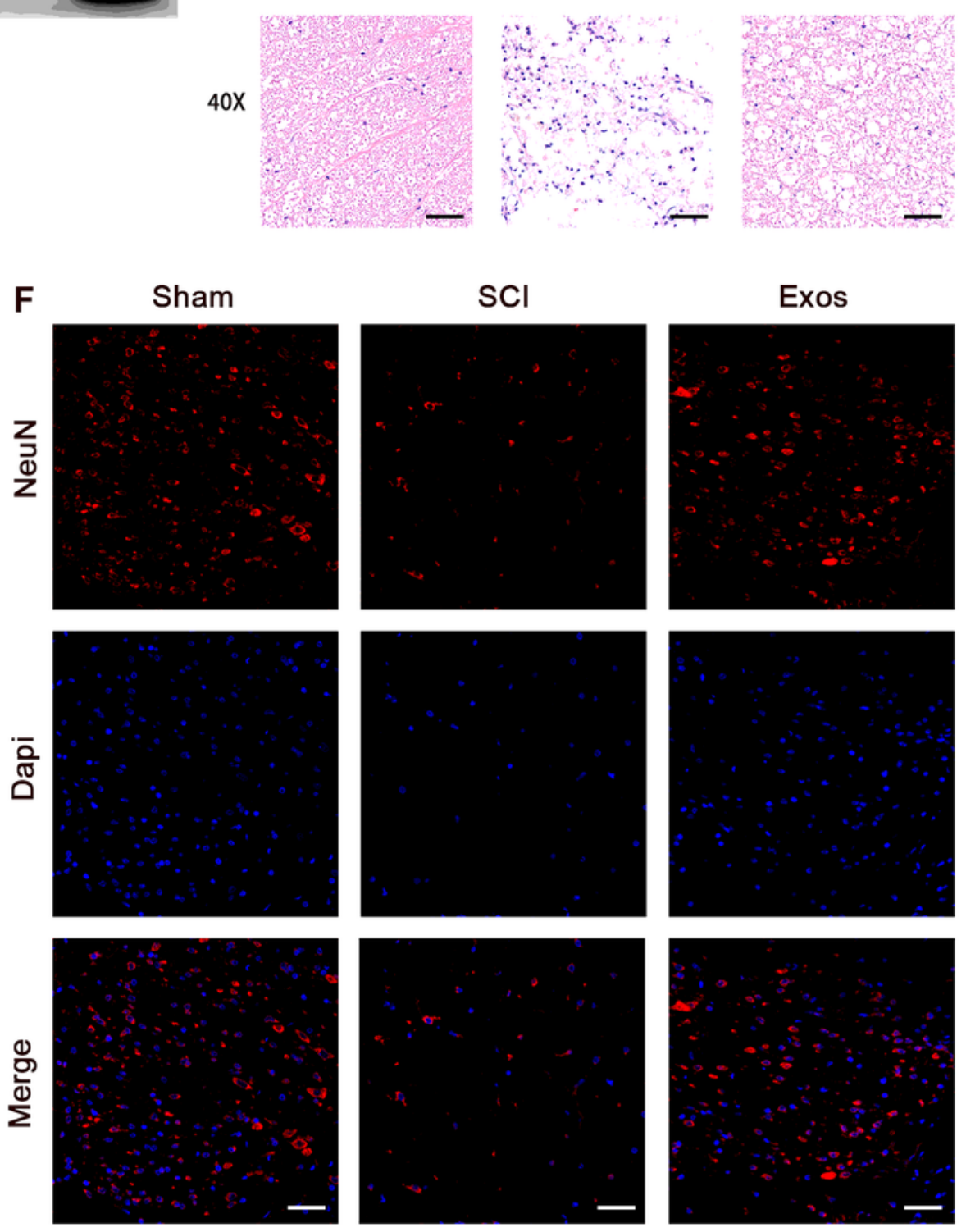

\section{Figure 1}

BMSC-Exos promotes SCl repair (A) qNano analysis results of BMSCs-Exos. (B) Western blotting showed the presence of exosomal markers including CD9, CD63, and TSG101 in BMSCs-Exos. (C) TEM photomicrographs of BMSCs-Exos. (D) The Basso, Beattie and Bresnahan (BBB) scores, *represents $\mathrm{P}<$ 0.05 versus the Sham group, \#represents $P<0.05$ versus the $S C l$ group $n=5$. (E) HE staining of 3 groups

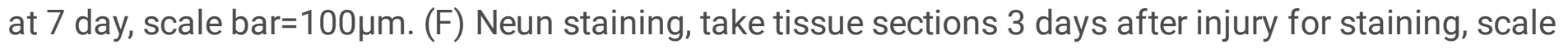


bar $=100 \mu \mathrm{m}$. (G) Statistics of neuron count, *represents $P<0.05$ versus the Sham group, \#represents $P<$ 0.05 versus the $\mathrm{SCl}$ group $\mathrm{n}=5$.

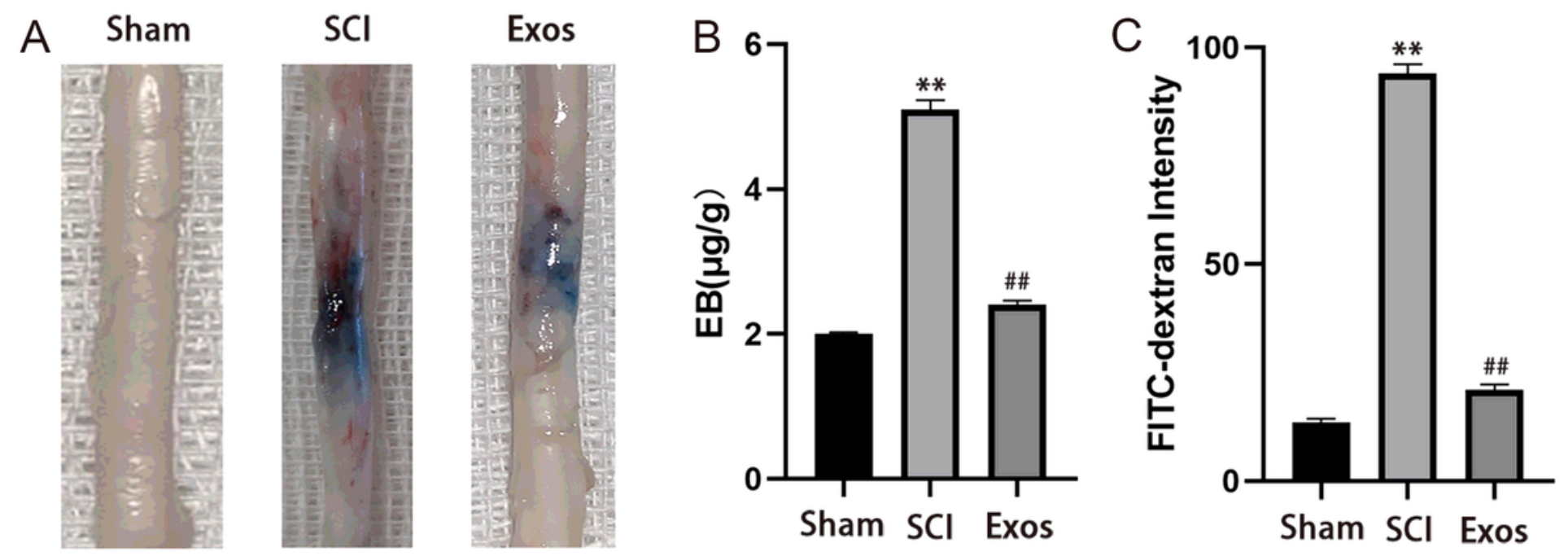

Figure 2

BMSC-Exos attenuates BSCB disruption to promote SCI repair (A) Representative spinal cords show that Evan's Blue dye permeabilized into injury spinal cord at 1 day ( $n=4 /$ group). (B) Quantification of the amount of Evan's Blue at 1 day $(\mu \mathrm{g} / \mathrm{g})$, **represents $P<0.01$ versus the Sham group, \#\#represents $P<$ 0.01 versus the $\mathrm{SCl}$ group $n=5$. (C) 1 day after spinal cord injury, FITC-dextran is in the spinal cord Peripheral penetration analysis results. **represents $\mathrm{P}<0.01$ versus the Sham group, \#\#represents $\mathrm{P}<$ 0.01 versus the $\mathrm{SCl}$ group $\mathrm{n}=5$. 

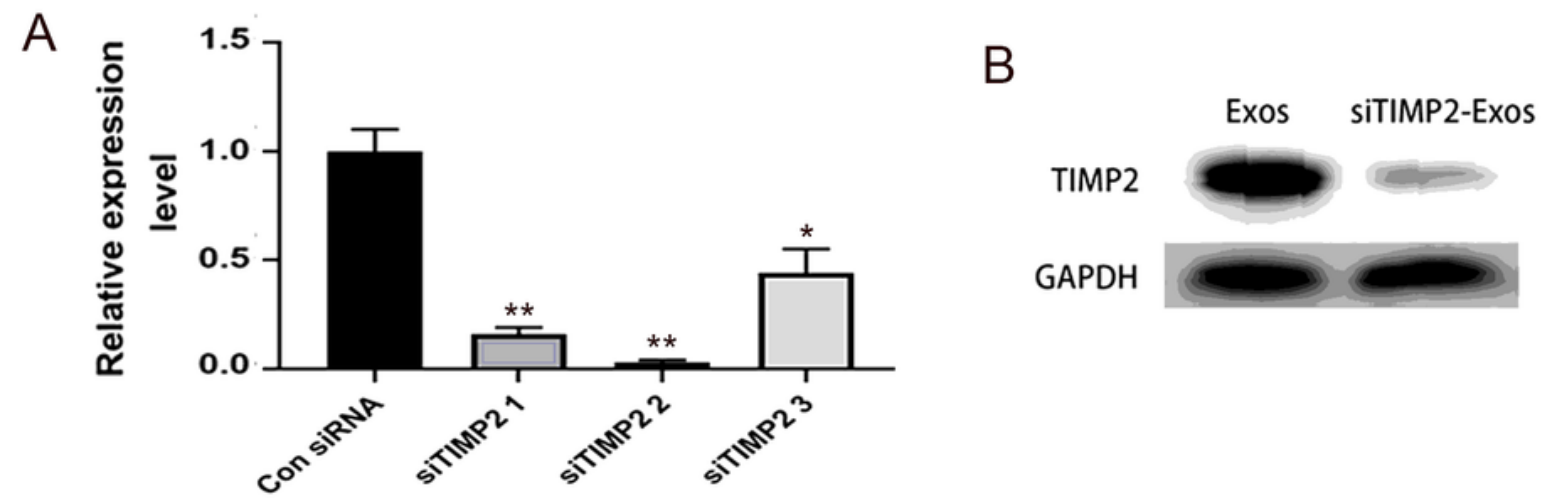

C

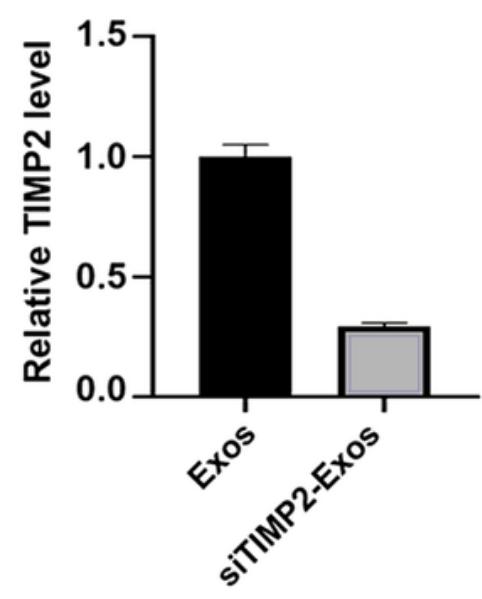

$E$

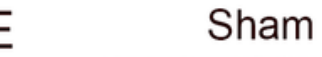

$10 X$
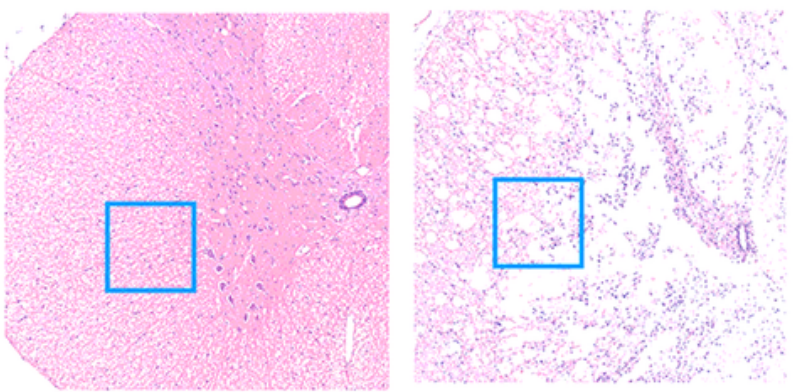

$40 \mathrm{X}$

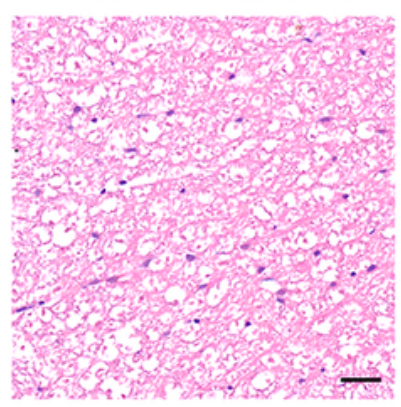

D

$\mathrm{SCl}$

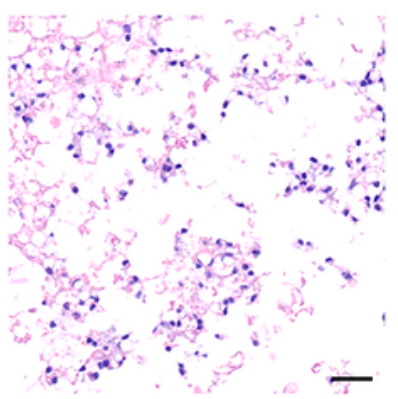

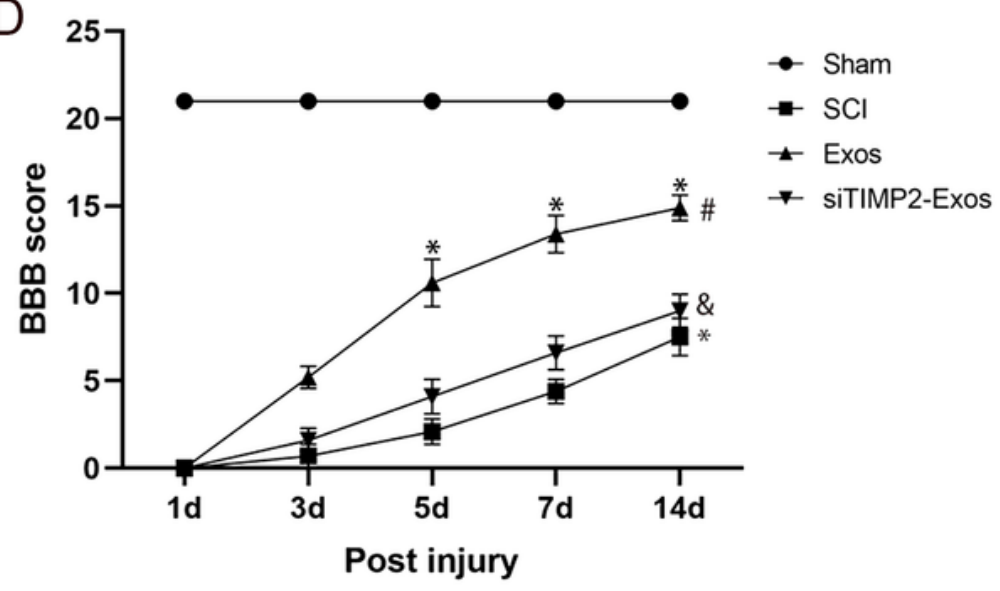

Exos

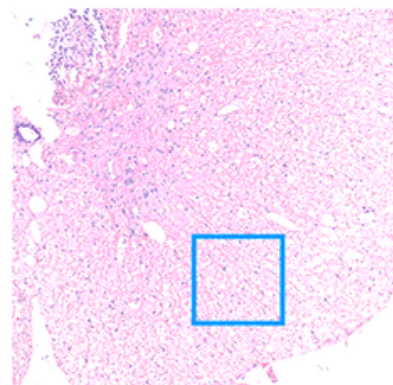

siTIMP2-Exos

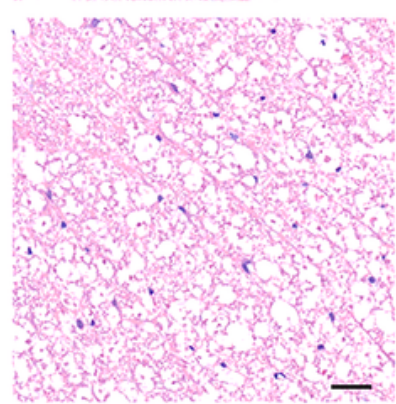

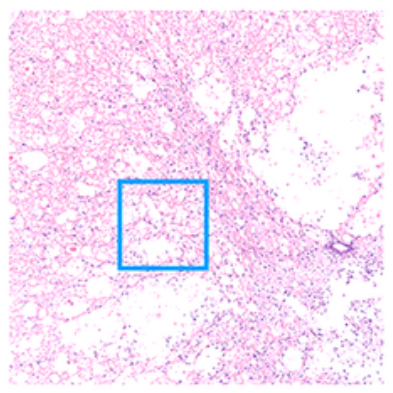

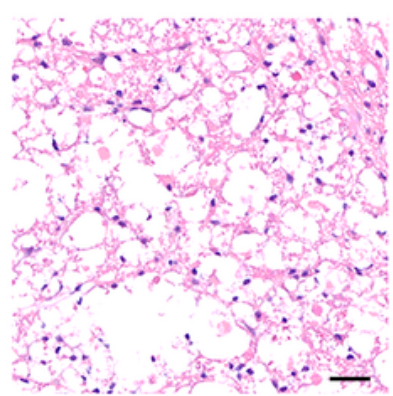

\section{Figure 3}

TIMP2 is involved in the effects of BMSC-Exos on SCl repair (A) The inhibitory efficiency of siRNAs targeting TIMP2 was verified by qRT-PCR analysis. *represents $\mathrm{P}<0.05$ vs. Con siRNA group, **represents $P<0.01$ vs. Con siRNA group $n=3$. (B and $C$ ) Western blot analysis of TIMP2 in exosomes from TIMP2silenced BMSCs (BMSCssiTIMP2 \#2-Exos) and from Con siRNA-treated BMSCs (BMSCsCon siRNA-Exos). 
represents $P<0.05$ versus the $S C l$ group, \& represents $P<0.05$ versus the Exos group, $n=5$. (E) HE staining of 4 groups at 7 day after SCl, scale bar=100 $\mu \mathrm{m}$.
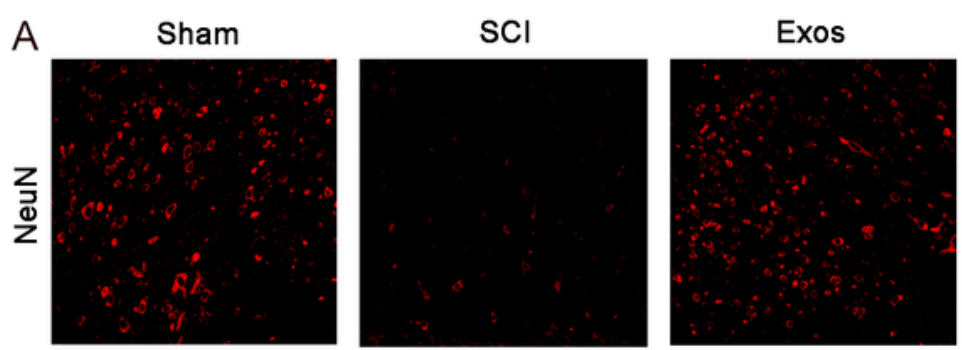

siTIMP2-Exos
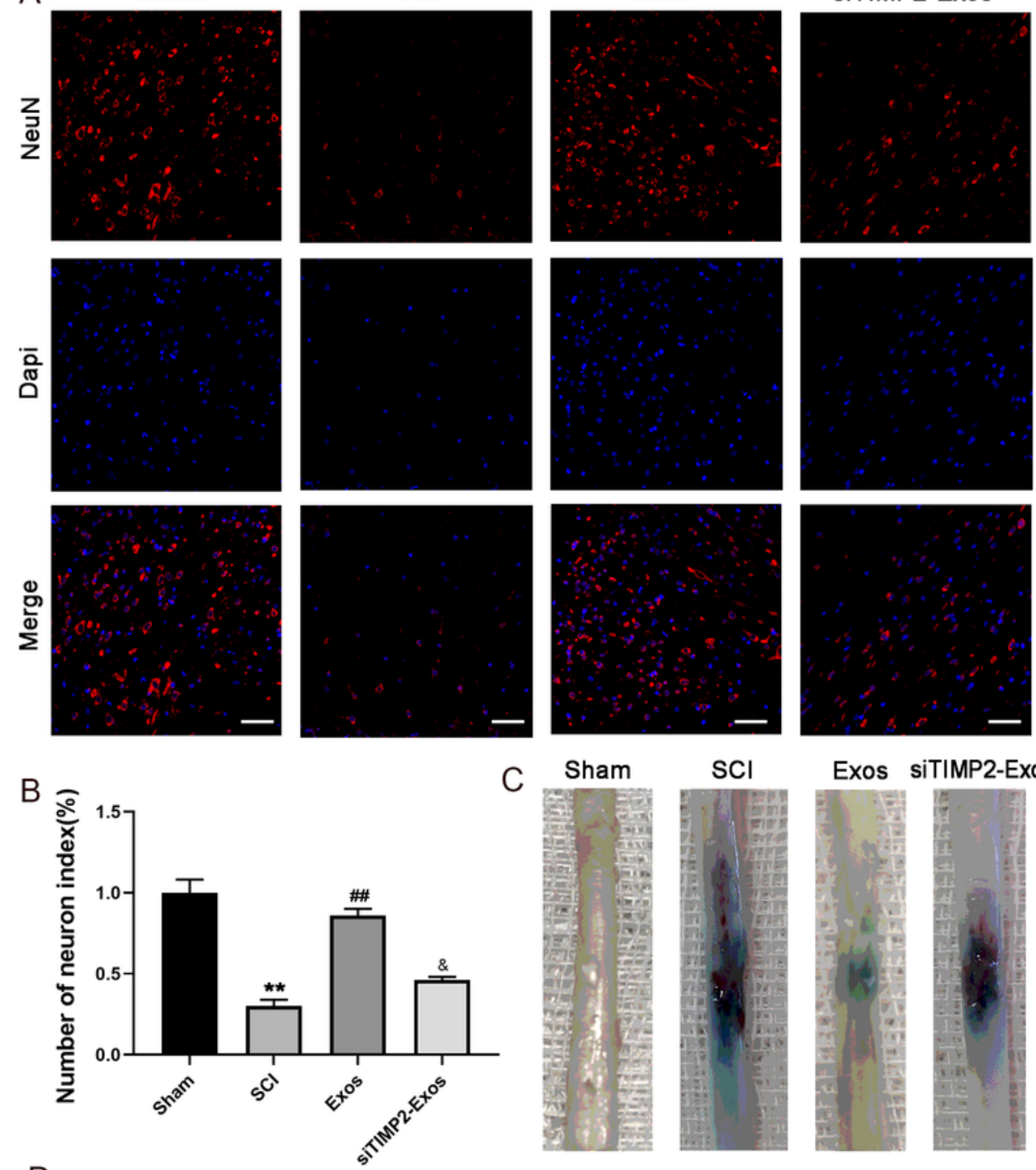

Exos siTIMP2-Exos
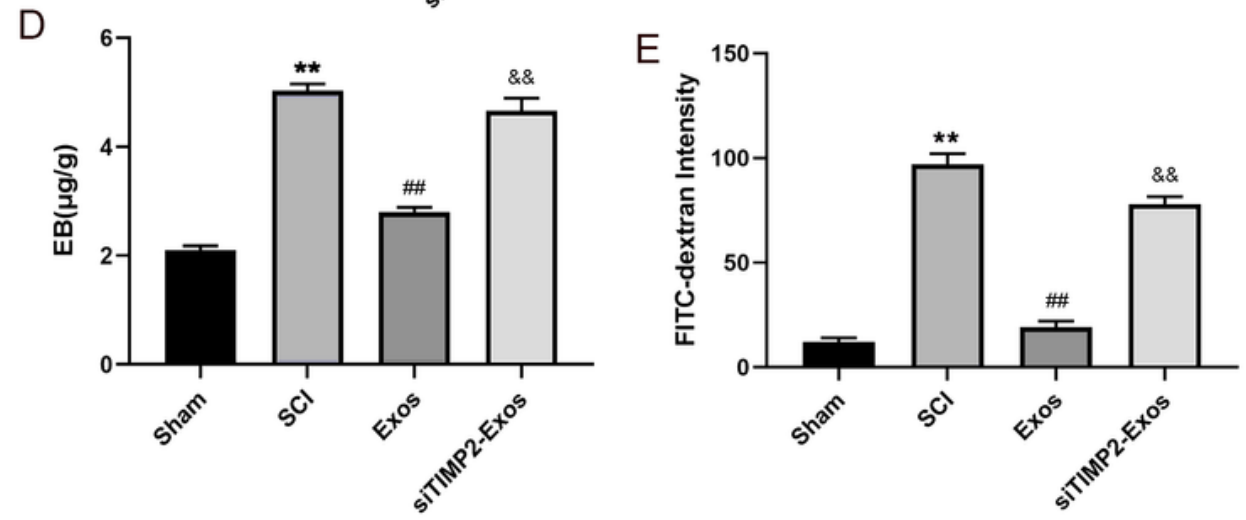

Figure 4

TIMP2 is involved in the effects of BMSC-Exos on SCI and BSCB repair (A) Neun staining, take tissue sections 3 days after $\mathrm{SCl}$ for staining, scale bar $=100 \mu \mathrm{m}$. (B) Statistics of neuron count, ** represents $\mathrm{P}<$ Loading [MathJax]/jax/output/CommonHTML/fonts/TeX/fontdata.js $s$ the $\mathrm{SCl}$ group, \& represents $\mathrm{P}<0.05$ versus 
the Exos group, $n=5$. (C) Representative spinal cords show that Evan's Blue dye permeabilized into injury spinal cord at 1 day ( $n=4 /$ group). (D) Quantification of the amount of Evan's Blue at 1 day $(\mu \mathrm{g} / \mathrm{g})$, ** represents $\mathrm{P}<0.01$ versus the Sham group, \#\# represents $\mathrm{P}<0.01$ versus the $\mathrm{SCl}$ group, \&\& represents $\mathrm{P}$ $<0.01$ versus the Exos group, $n=5$ (E) 1 day after spinal cord injury, FITC-dextran is in the spinal cord Peripheral penetration analysis results. **represents $\mathrm{P}<0.01$ versus the Sham group, \#\#represents $\mathrm{P}<$ 0.01 versus the $\mathrm{SCl}$ group, \&\& represents $\mathrm{P}<0.01$ versus the Exos group, $\mathrm{n}=5$.

A Sham SCl Exos siTIMP2-Exos

TIMP2

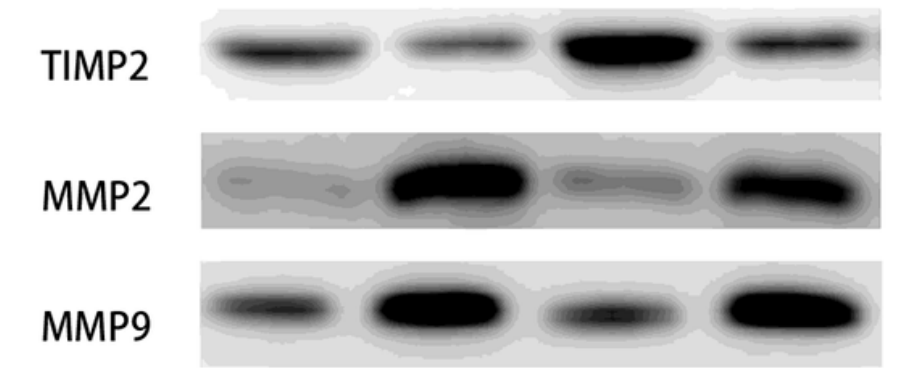

Claudin5

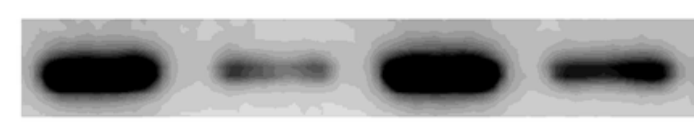

occludin

$B$
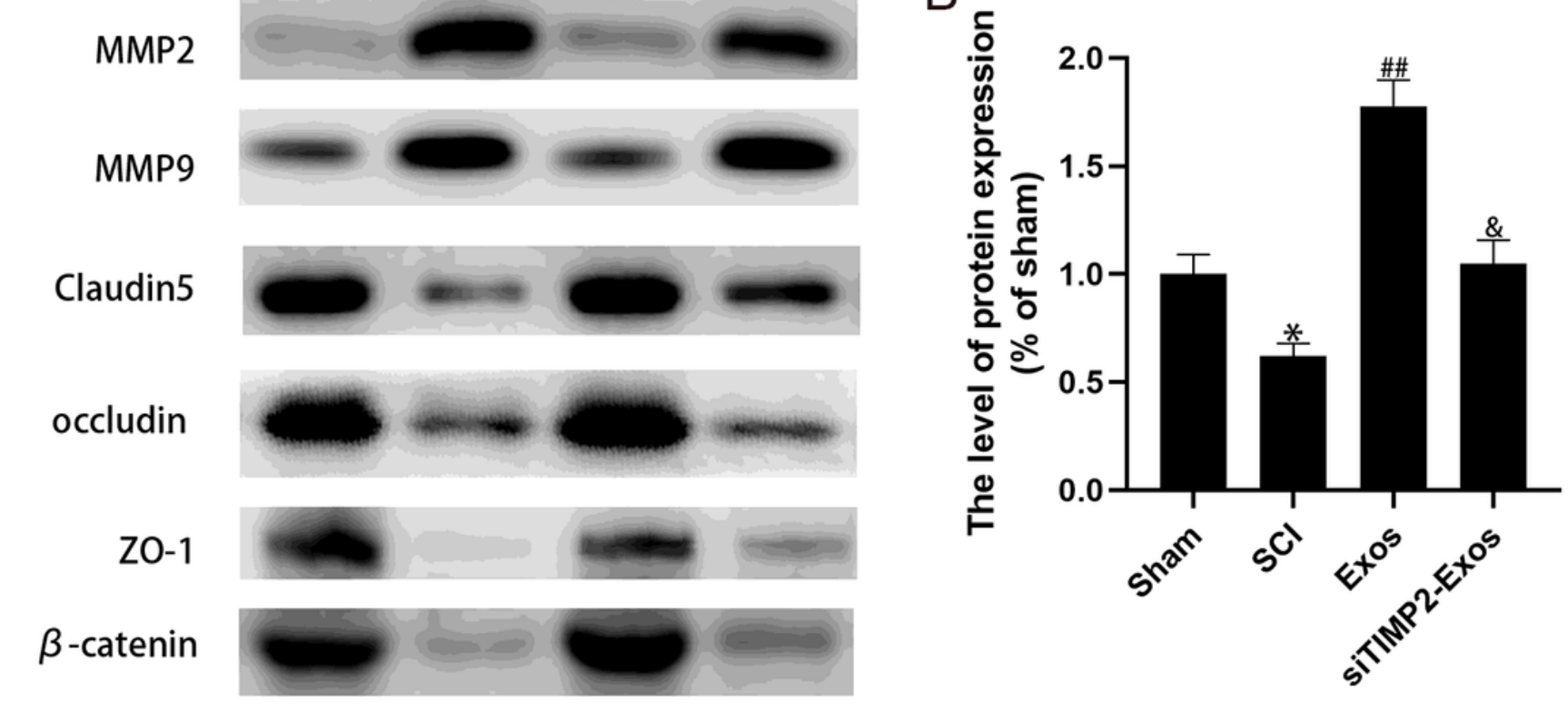

GAPDH
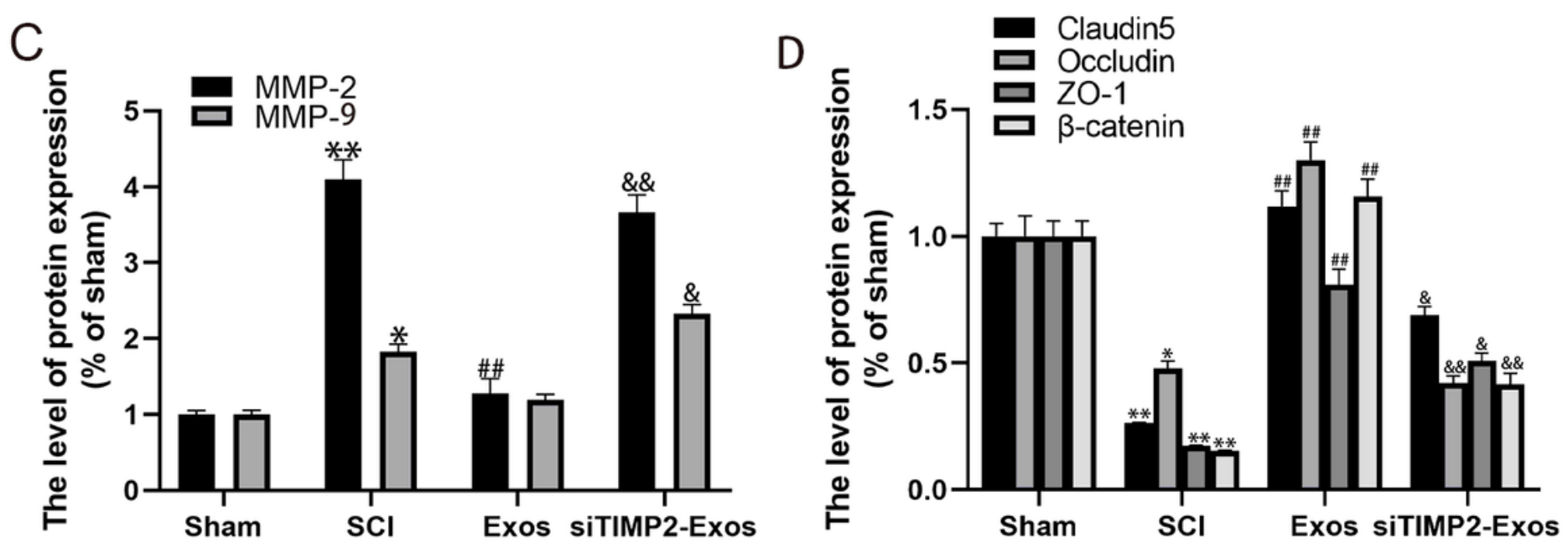

Figure 5

BMSC-Exos increases the expression of junction proteins after SCI via TIMP2/MMP pathway (A-D) Loading [MathJax]/jax/output/CommonHTML/fonts/TeX/fontdata.js and B-catenin, MMP-2, MMP-9 in spinal cord of 
Sham, SCl, Exos, siTIMP2-Exos groups 1 day after SCl. *represents $\mathrm{P}<0.05$ versus the Sham group, **represents $\mathrm{P}<0.01$ versus the Sham group, \#\#represents $\mathrm{P}<0.01$ versus the $\mathrm{SCl}$ group, \& represents $\mathrm{P}$ $<0.05$ versus the Exos group, \&\& represents $P<0.01$ versus the Exos group, $n=5$.
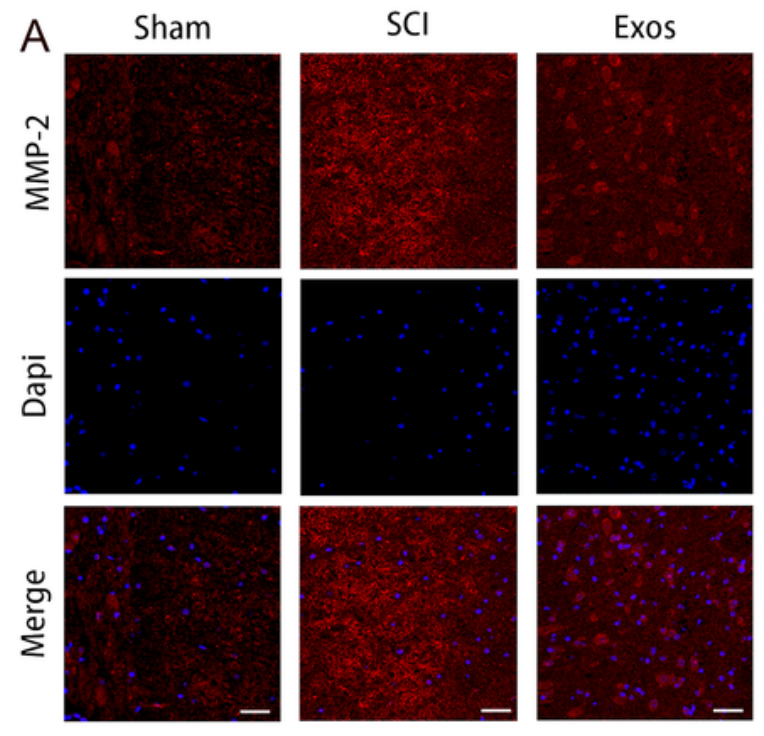

SITIMP2-Exos
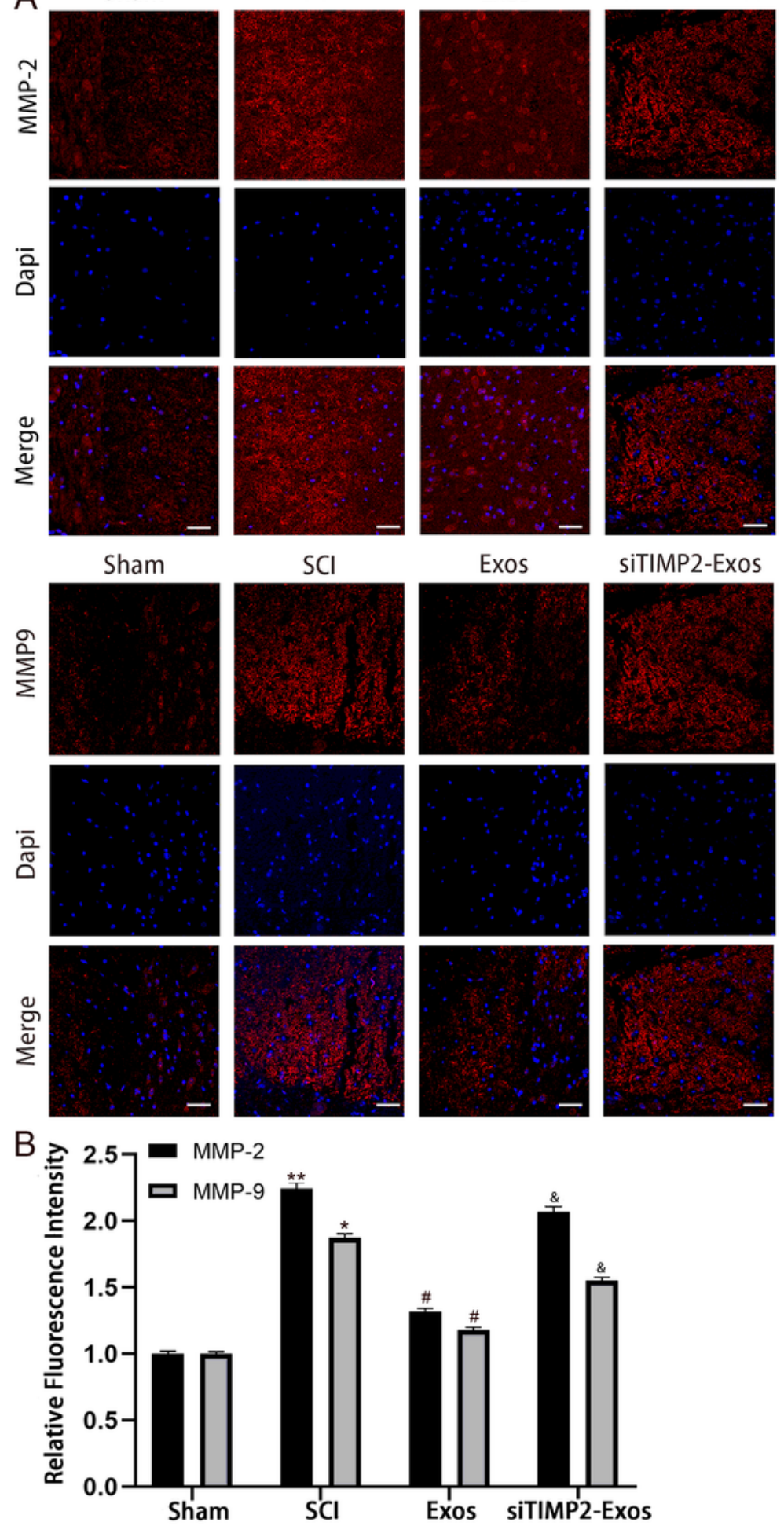

Figure 6

BMSC-Exos increases the expression of junction proteins after SCI via TIMP2/MMP pathway (the results Loading [MathJax]/jax/output/CommonHTML/fonts/TeX/fontdata.js staining of MMP-2 and MMP-9 in spinal cord of 
the above four groups 1 day after $\mathrm{SCl}$. *represents $\mathrm{P}<0.05$ versus the Sham group, ** represents $\mathrm{P}<0.01$ versus the Sham group, \& represents $P<0.05$ versus the Exos group, $n=5$, scale bar $=100 \mu m$

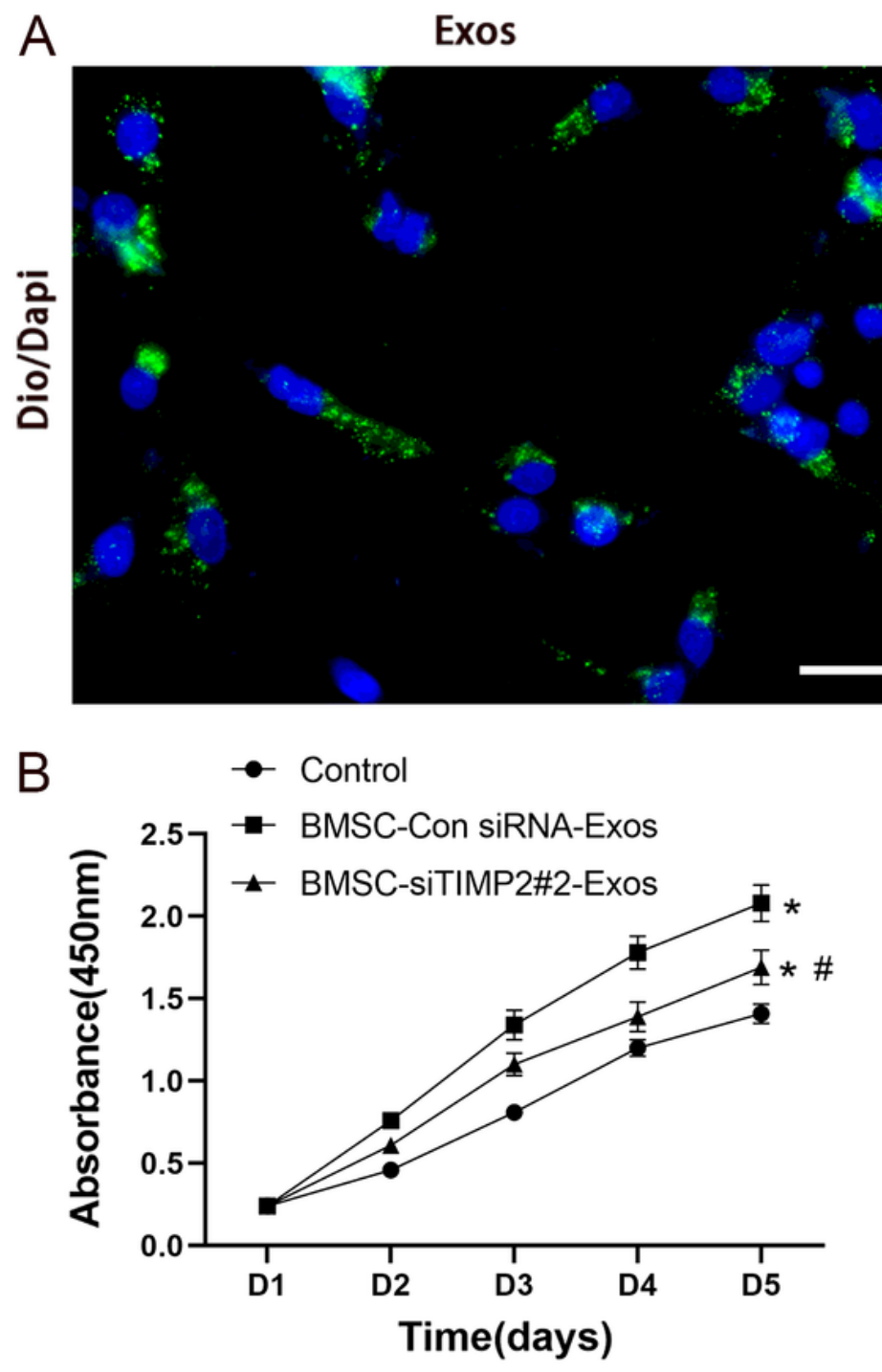

siTIMP2-Exos

$\mathrm{D}$

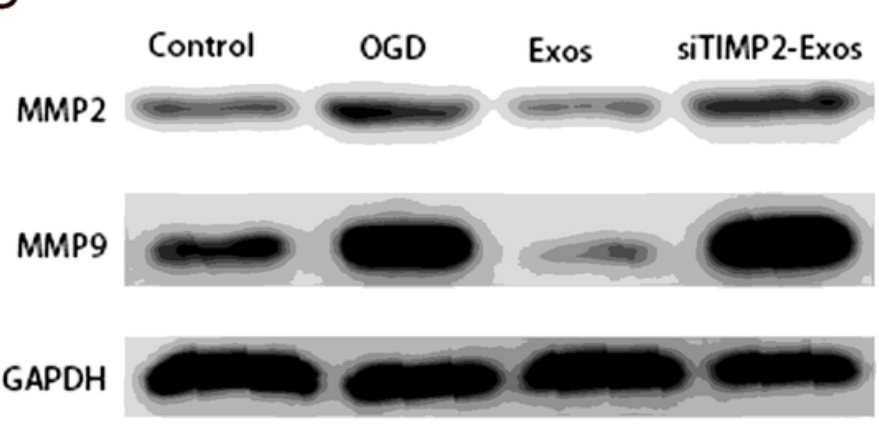

C

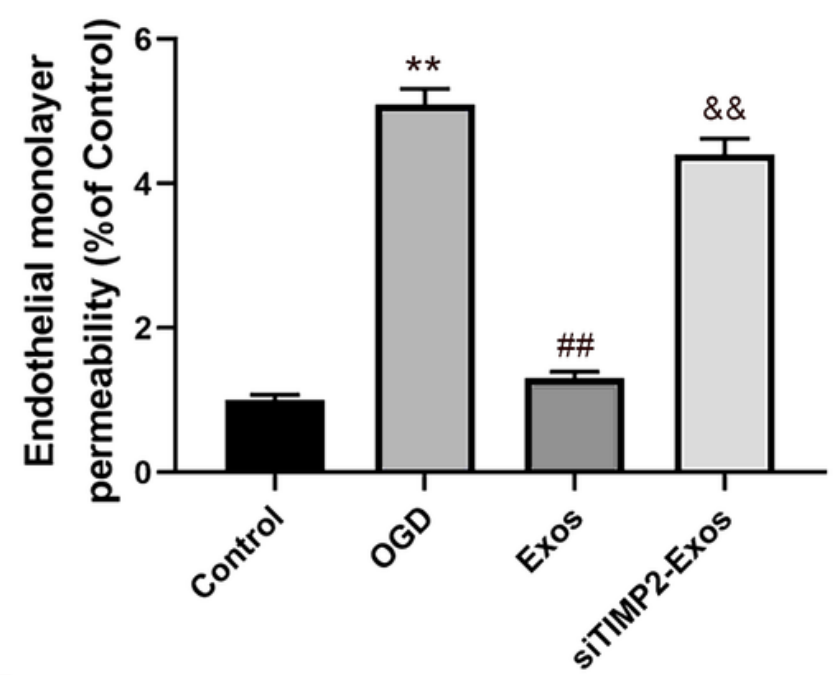

$\mathrm{E}$

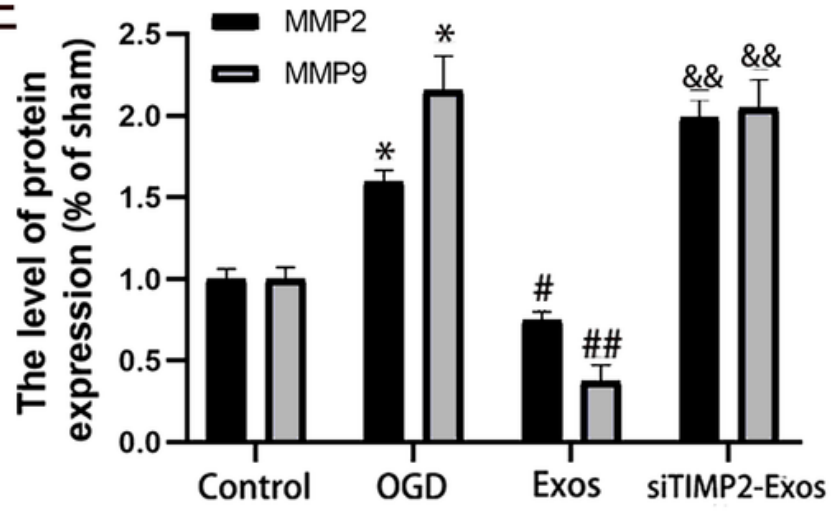

Figure 7

The effects of BMSC-Exos on junction proteins are inhibited by siTIMP2 in endothelial cells after oxygenglucose deprivation (A) Fluorescent microscopy showed the internalization of DIO-labeled Exos of SiTIMP2-Exos by HBMECs. Exosomes could be seen in the perinuclear region of HBMECs, scale 
bar $=50 \mu \mathrm{m}$. (B) The proliferation of HBMECs treated with PBS, BMSCs-Con siRNA-Exos and BMSCssiTIMP2 \#2-Exos was tested by CCK-8 analysis, *represents $P<0.05$ versus the $P B S$ group, \# represents $P$ $<0.05$ versus the BMSCs-Con siRNA-Exos group, $n=5$. (C) Under different conditions, FITC-dextran permeates the fluorescence intensity of the lower chamber. **represents $\mathrm{P}<0.01$ versus the CON group, $\mathrm{n}=5$. (D and E) Western blot analysis of MMP-2, MMP-9 in control, OGD, Exos, siTIMP2-Exos groups, *represents $P<0.05$ versus the CON group, \#represents $P<0.05$ versus the OGD group, \#\#represents $P<$ 0.01 versus the OGD group, \&\& represents $P<0.01$ versus the Exos group, $n=5$.
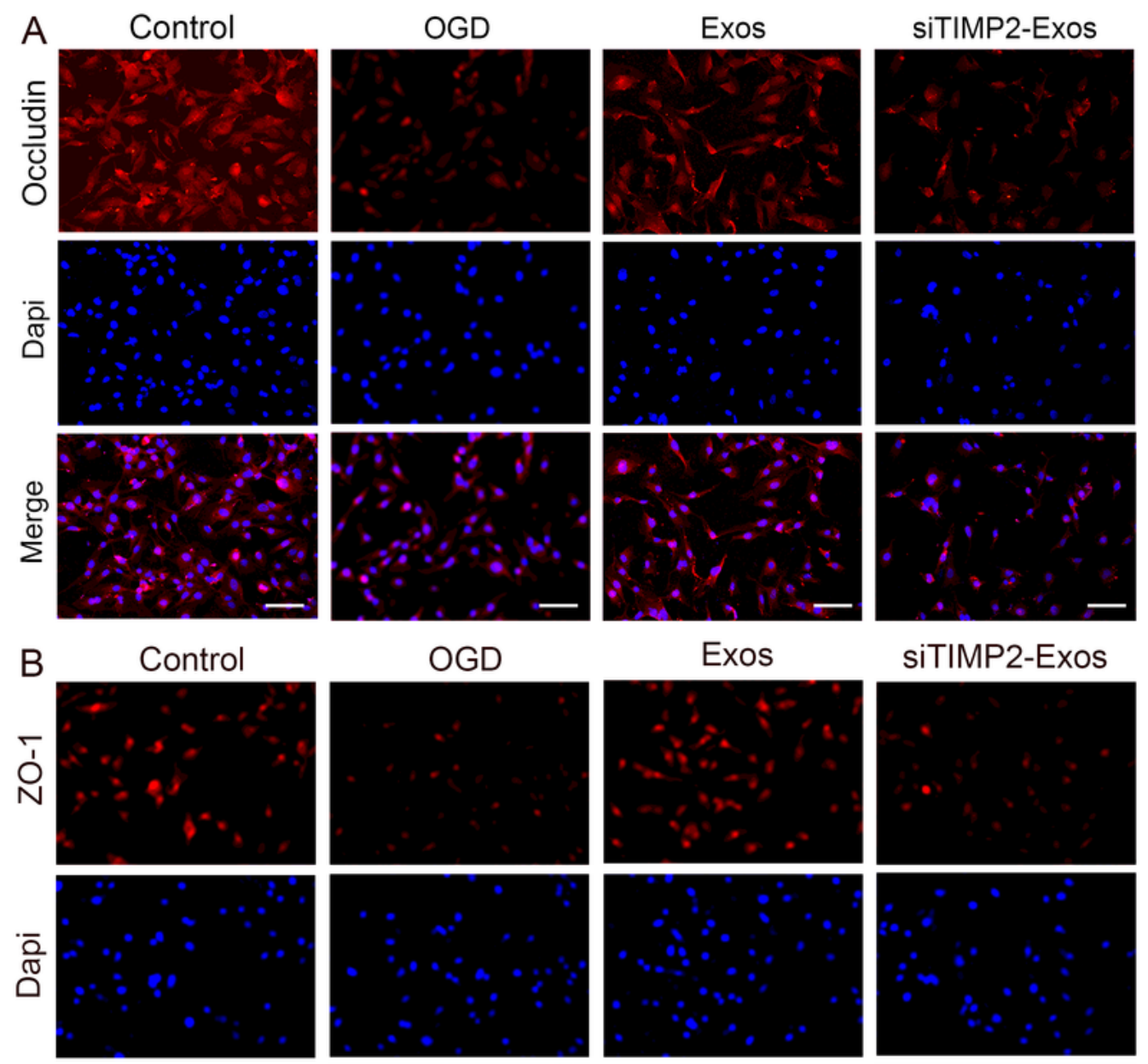

siTIMP2-Exos
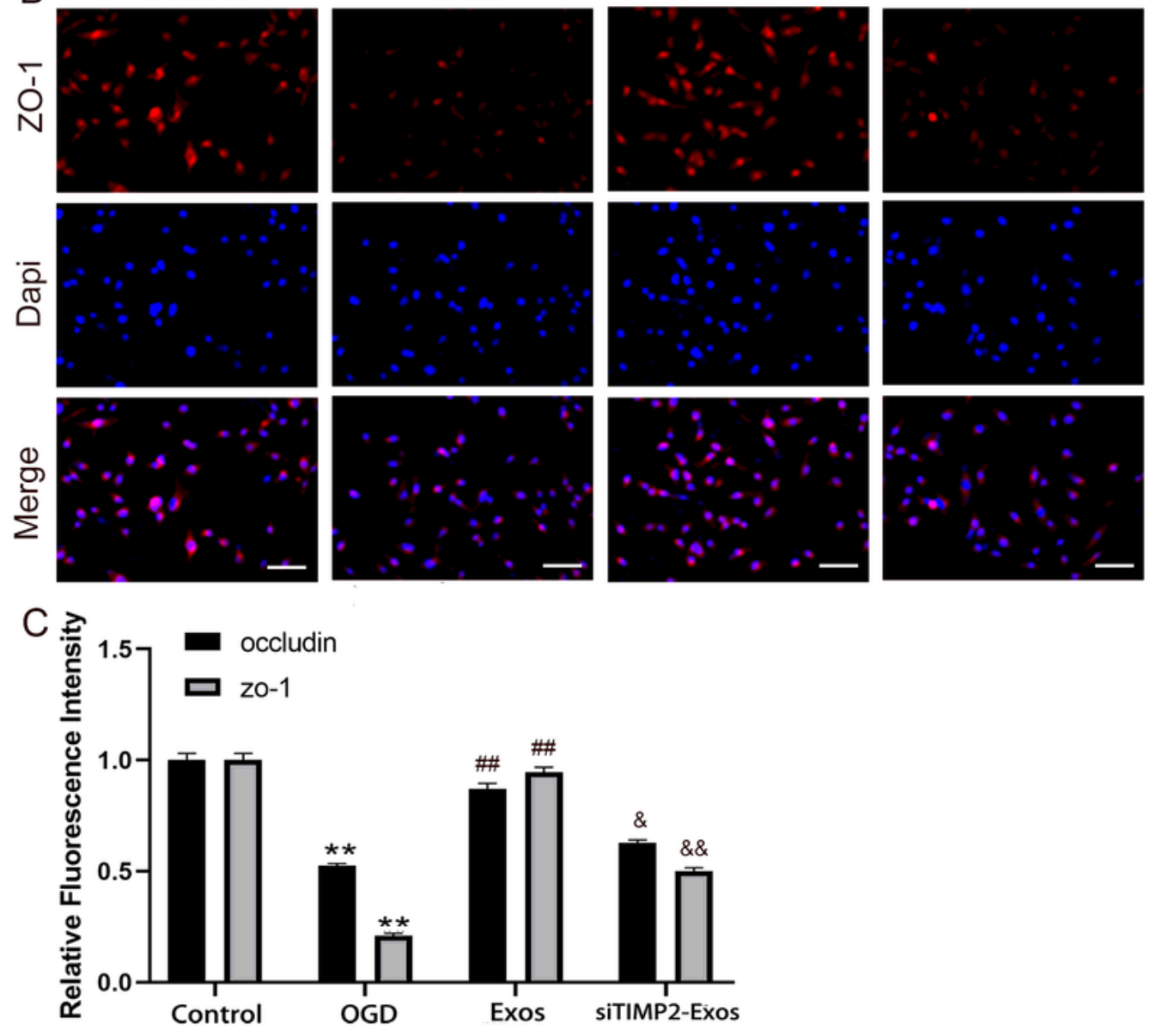

Loading [MathJax]/jax/output/CommonHTML/fonts/TeX/fontdata.js 


\section{Figure 8}

The effects of BMSC-Exos on junction proteins are inhibited by siTIMP2 in endothelial cells after oxygenglucose deprivation (the results of Immunofluorescence). (A-C) Immunofluorescence staining of occluding and zo-1 in HBMECs of the above four groups, **represents $\mathrm{P}<0.05$ versus the CON group, \#\#represents $\mathrm{P}<0.01$ versus the OGD group, \& represents $\mathrm{P}<0.05$ versus the Exos group, \&\& represents $P<0.01$ versus the Exos group, $n=5$, scale bar $=50 \mu m$. 